\title{
Differential Peroxiredoxin Hyperoxidation Regulates MAP Kinase Signaling in Human Articular Chondrocytes
}

\author{
John A. Collins ${ }^{1}$, Scott T. Wood ${ }^{1,2}$, Jesalyn A. Bolduc ${ }^{1}$, N.P. Dewi Nurmalasari ${ }^{2}$, Susan \\ Chubinskaya $^{3}$, Leslie B. Poole ${ }^{4}$, Cristina M. Furdui ${ }^{5}$, Kimberly J. Nelson ${ }^{4}$, and Richard F. \\ Loeser $^{1}$ \\ ${ }^{1}$ Division of Rheumatology, Allergy and Immunology and the Thurston Arthritis Research Center, \\ University of North Carolina at Chapel Hill, Chapel Hill, NC, USA. \\ ${ }^{2}$ Nanoscience and Nanoengineering, South Dakota School of Mines and Technology, BioSNTR, \\ Rapid City, SD, USA. \\ ${ }^{3}$ Department of Pediatrics, Rush University Medical Center, Chicago, IL, USA. \\ ${ }^{4}$ Department of Biochemistry, Wake Forest School of Medicine, Winston-Salem, NC, USA. \\ ${ }^{5}$ Department of Internal Medicine, Section on Molecular Medicine, Wake Forest School of \\ Medicine, Winston-Salem, NC, USA.
}

\section{Abstract}

The peroxiredoxin (Prx) family of Cys-dependent peroxidases control intracellular levels of $\mathrm{H}_{2} \mathrm{O}_{2}$ and can regulate signal transduction. Inhibition of the Prxs, through hyperoxidation amongst other mechanisms, leads to oxidative stress conditions that can alter homeostatic signaling. To determine the effects oxidation of Prx1-Prx3 has on MAP kinase and IGF-1 signaling events in human chondrocytes, this study used 2-methyl-1,4-naphthoquinone (menadione) and 2,3-dimethyl-1,4naphthoquinone (DMNQ) as $\mathrm{H}_{2} \mathrm{O}_{2}$ generating tools due to their differential mechanisms of action. Menadione and DMNQ generated similar levels of intracellular $\mathrm{H}_{2} \mathrm{O}_{2}$ as determined using the biosensor Orp1-roGFP and by measuring Prx redox status. However, menadione generated higher levels of mitochondrial $\mathrm{H}_{2} \mathrm{O}_{2}$ associated with Prx3 hyperoxidation and phosphorylation of Prx 1 while DMNQ treatment was associated with hyperoxidation of cytosolic Prx1 and Prx2 but not mitochondrial Prx3. Both menadione and DMNQ induced sustained phosphorylation of p38 but only DMNQ activated JNK. Menadione but not DMNQ inhibited IGF-1induced Akt phosphorylation. Chondrocytes transduced with an adenoviral vector to overexpress Prx3 displayed decreased $\mathrm{PrxSO}_{2 / 3}$ formation in response to menadione which was associated with restoration of IGF-1-mediated Akt signaling and inhibition of p38 phosphorylation. Prx1 and Prx2 overexpression had no effects on Prx redox status but Prx 1 overexpression enhanced basal Akt

Corresponding author: Richard F. Loeser, Thurston Arthritis Research Center, The University of North Carolina at Chapel Hill, 3300 Thurston Building, Campus Box 7280, Chapel Hill, NC, 27599-7280, richard_loeser@med.unc.edu.

Conflict of interest: The authors declare that they have no conflicts of interest with the contents of this article.

Publisher's Disclaimer: This is a PDF file of an unedited manuscript that has been accepted for publication. As a service to our customers we are providing this early version of the manuscript. The manuscript will undergo copyediting, typesetting, and review of the resulting proof before it is published in its final citable form. Please note that during the production process errors may be discovered which could affect the content, and all legal disclaimers that apply to the journal pertain. 
phosphorylation. These results suggest that hyperoxidation of specific Prx isoforms is associated with distinct cell signaling events and identify Prx3 redox status as an important regulator of anabolic and catabolic signal transduction. Targeted strategies to prevent mitochondrial Prx3 hyperoxidation could be useful in maintaining cellular redox balance and homeostatic signaling.

\section{Graphical Abstract}

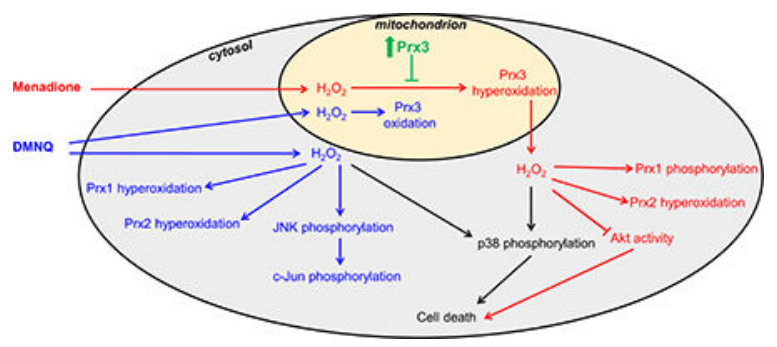

\section{Keywords}

Peroxiredoxin; oxidative stress; redox signaling; cartilage; MAP kinase signaling; osteoarthritis

\section{Introduction}

Through its controlled release, $\mathrm{H}_{2} \mathrm{O}_{2}$ acts as a key second messenger in specific signaling pathways that are important for cellular homeostasis. The balance between reactive oxygen species (ROS) production and subsequent elimination via intracellular antioxidant defence systems is crucial in maintaining redox balance (1). If overproduced or over-accumulated, ROS, such as $\mathrm{H}_{2} \mathrm{O}_{2}$, can cause cellular dysfunction by inflicting non-discriminatory damage to lipids, proteins, and DNA or through disturbing physiological cell signaling events (2-4). Although our understanding of how ROS regulate cell signaling is far from complete, posttranslational oxidative modifications on reactive protein cysteines is now viewed as an important mechanism capable of regulating intracellular signal transduction $(2,5)$.

The peroxiredoxins (Prxs) are a family of cysteine-dependent peroxidases that react rapidly with $\mathrm{H}_{2} \mathrm{O}_{2}$. As a result, these are considered to be the major antioxidant enzymes that contribute to cellular redox state (6-8). To catalytically breakdown $\mathrm{H}_{2} \mathrm{O}_{2}$, the sulfhydryl group of the Prx peroxidatic cysteine undergoes oxidation to form sulfenic acid (Prx-SOH), a process termed sulfenylation. For typical 2-Cys Prxs, the peroxidatic cysteine can condense with a Prx resolving cysteine to form an inter-subunit disulfide. The disulfide bond can be reduced back by the thioredoxin reductase system to complete the Prx catalytic cycle, restoring the Prx back to its catalytically active state $(4,8)$. Under increasing levels of $\mathrm{H}_{2} \mathrm{O}_{2}$, instead of disulfide formation, Prxs can react with additional molecules of $\mathrm{H}_{2} \mathrm{O}_{2}$ and become hyperoxidized to form sulfinic $\left(\mathrm{Prx}-\mathrm{SO}_{2} \mathrm{H}\right)$ and sulfonic acid $\left(\mathrm{Prx}-\mathrm{SO}_{3} \mathrm{H}\right)$, resulting in loss of peroxidase activity. Hyperoxidized Prxs, at least when sulfinylated but not sulfonylated, can be generally repaired back to their active, reduced form by sulfiredoxin, but this is a slow process which also requires ATP (9). 
As a result of their cellular abundance and the high reactivity of the peroxidatic cysteine with $\mathrm{H}_{2} \mathrm{O}_{2}$, Prxs are considered both sensors and preferential targets of $\mathrm{H}_{2} \mathrm{O}_{2}$, but their roles extend beyond that of an antioxidant enzyme $(8,10)$. Inhibition of Prx peroxidase activity by hyperoxidation is no longer just viewed as a deleterious endpoint in response to high levels of ROS. In fact, it may be a conserved mechanism to protect thioredoxin levels under conditions of oxidative stress (11), promote Prx chaperone activity (11), and/or regulate $\mathrm{H}_{2} \mathrm{O}_{2}$-induced cell signal transduction (7). Phosphorylation of Prx1 on Tyr194 has also been shown to inhibit Prx activity which, when compared to hyperoxidation, may represent a more transient and reversible posttranslational modification that can regulate local $\mathrm{H}_{2} \mathrm{O}_{2}$ mediated cell signaling (12). Alternatively, Prxs can participate in thiol-disulfide exchange, whereby a sulfenylated Prx transfers its oxidizing equivalent to a nearby redox sensitive protein, forming a redox relay for signal transduction, as has been shown for Prx 2 and the transcription factor STAT3 (13). Collectively, these lines of evidence suggest that alterations in Prx redox status are an important intermediary step in $\mathrm{H}_{2} \mathrm{O}_{2}$-mediated signal transduction $(8,10)$ but there is a paucity of data detailing the role of Prx redox status and associated cell signaling in human primary cells.

Importantly, different cellular compartments contain different ROS generating machinery, and in turn, express different antioxidant enzymes in order to respond to changes in ROS production. In humans, four typical 2 Cys-Prxs (Prx1-Prx4), one atypical 2 Cys-Prx (Prx5) and a 1 Cys-Prx (Prx6) are involved in redox signaling and each Prx isoform displays specific subcellular compartmentalization. Prx1, Prx2 and Prx6 are predominantly located in the cytosol, Prx3 is located in the mitochondria, Prx4 is located in the endoplasmic reticulum and Prx5 has been observed in the cytosol, mitochondria and in peroxisomes (14). It has been demonstrated that Prx isoforms display different susceptibilities to hyperoxidation (15-17). Mitochondrial Prx3 has been shown to display an increased resistance to hyperoxidation when compared to cytosolic Prx1 and Prx2 (15-17), which could suggest that differential $\mathrm{Prx}$ hyperoxidation is a mechanism to regulate $\mathrm{H}_{2} \mathrm{O}_{2}$ levels within distinct cellular compartments in order to regulate specific cell signaling events.

While conducting experiments treating human chondrocytes with two different redox cycling, $\mathrm{H}_{2} \mathrm{O}_{2}$-generating compounds, menadione and DMNQ, we noted differential hyperoxidation of Prx1-Prx3 specific to each compound. We used this observation to test the hypothesis that hyperoxidation of different Prx isoforms, residing in different cellular compartments, would lead to different effects on redox-regulated cell signaling events. Menadione and DMNQ were therefore used as $\mathrm{H}_{2} \mathrm{O}_{2}$-generating tools in order to investigate the role of Prx hyperoxidation on selected anabolic and catabolic signaling pathways. These studies were conducted in primary cultures of human articular chondrocytes as a relevant cell type due to work showing that oxidative stress disrupts cell signaling in chondrocytes resulting in reduced anabolic and increased catabolic activity as well as increased cell death (18). 


\section{Materials and methods}

\subsection{Antibodies and reagents}

Antibodies purchased from Cell Signaling Technology were to phospho-IGF receptor (Tyr1135), totalIGF receptor, phospho-Akt (Ser473), total-Akt, phospho-PRAS40 (Thr246), total PRAS40, phospho-p38 (Thr180/Tyr182), total-p38, phospho-ERK (Thr202/Tyr204), total-ERK, phospho-JNK (Thr183/Tyr185), total JNK, phospho c-Jun (ser73), total c-Jun, phospho-Prx 1 (Tyr194), total-Prx1 and $\beta$-tubulin. Antibodies purchased from Abcam were to $\operatorname{PrxSO}_{2 / 3}$, Prx2, Prx3 and $\beta$-actin. The Orp1-roGFP cellular hydrogen peroxide sensor $\left(\right.$ Premo ${ }^{\mathrm{TM}}$ ) was purchased from Life Technologies. Menadione, DMNQ, Nethylmaleimide (NEM) and catalase were purchased from Sigma Aldrich.

Human primary chondrocyte isolation and culture.-Normal articular cartilage was obtained from the talus of human tissue donors provided by the Gift of Hope Organ and Tissue Donor Network (Itasca, IL) through collaboration with Rush University Medical Center (Chicago, IL). Prior to dissection, cartilage was macroscopically inspected for gross evidence of damage and only normal appearing cartilage was used. The ages of tissue donors ranged from 38 to 71 years (mean age $\pm \mathrm{SD}, 58.7 \pm 9.5$ years). Chondrocytes were isolated and cultured in monolayer as previously described (19).

\subsection{Adenoviral transduction}

Human chondrocytes were transduced with adenoviral constructs encoding Prx1 (ad-Prx1), Prx2 (adPrx2) or Prx3 (ad-Prx3) (Vector Biolabs). On achievement of 60\% confluency, chondrocyte monolayers were incubated for $2 \mathrm{~h}$ at $37^{\circ} \mathrm{C}$ in serum-free media containing the adenoviral construct $\left(4 \times 10^{8}\right.$ viral particles $\left./ \mathrm{mL}\right)$ and $\mathrm{CaCl}_{2}(25 \mu \mathrm{L} / \mathrm{mL}$ media, $1 \mathrm{M})$.

Adenoviral containing media was removed, monolayers were rinsed three times with $10 \%$ serum containing media and monolayers were cultured in $10 \%$ serum containing media for $48 \mathrm{~h}$. Chondrocytes cultures were incubated overnight in serum-free media prior to experimental treatments. Non-specific effects of viral transduction were tested by transducing chondrocytes with a null empty vector control adenovirus (ad-Null) (Cell Biolabs).

\subsection{Quantification of intracellular $\mathrm{H}_{2} \mathrm{O}_{2}$ production}

We have previously demonstrated the effects of menadione to generate intracellular $\mathrm{H}_{2} \mathrm{O}_{2}$ in human chondrocytes (18). DMNQ has also been shown to induce cellular ROS in various tissues and cells through a similar mechanism (20). Intracellular $\mathrm{H}_{2} \mathrm{O}_{2}$ generation was quantified using the hydrogen peroxide sensor, Orp1-roGFP. Human chondrocyte cell culture, baculovirus transduction, live cell image acquisition and analysis were performed as previously described (18). Images were collected every $10 \mathrm{~s}$ for $1 \mathrm{~min}$ prior to experimental treatments to obtain basal $\mathrm{H}_{2} \mathrm{O}_{2}$ levels. Menadione $(25 \mu \mathrm{M})$ or DMNQ $(25 \mu \mathrm{M})$ were then added to chondrocyte cultures and cells were imaged every $10 \mathrm{~s}$ for a total of $2 \mathrm{~min}$ (18). Analysis of images was conducted in ImageJ using the FIJI plugin, and the sensitized FRET method (21) as previously described (18). 


\subsection{Quantification of mitochondrial $\mathrm{H}_{2} \mathrm{O}_{2}$ production}

Mitochondrial $\mathrm{H}_{2} \mathrm{O}_{2}$ generation was quantified using the adeno-mito-Orp1-roGFP redox biosensor, which was a kind gift from Dr. Brian O'Rourke, The Johns Hopkins University, Baltimore, MD, USA. Human chondrocytes were cultured in $35 \mathrm{~mm}$ imaging dishes (World Precision Instruments) for 5 days in Dulbecco's Modified Eagles Media (DMEM)/F12 supplemented with $10 \%$ ascorbic acid phosphate. To allow for chondrocyte adhesion, imaging dishes were plasma etched (Harrick Plasma) for 3 mins then immediately coated with $1 \mu \mathrm{g} / \mathrm{mL}$ of human type VI collagen (Rockland) for $2 \mathrm{~h}$ at $37{ }^{\circ} \mathrm{C}$ and washed with PBS prior to cell seeding. Chondrocytes were transduced with the adenovirus expressing mitoOrp1roGFP $\left(4 \times 10^{8}\right.$ viral particles $\left./ \mathrm{mL}\right)$ for $48 \mathrm{~h}$. Cells were serum starved for $2 \mathrm{~h}$ prior to imaging and imaged using oblique single plane illumination microscopy (oSPIM; Applied Scientific Instrumentation). Light sheets were generated at an oblique angle using a 100x 1.49 NA oil total internal reflection fluorescence (TIRF) objective. Fluorescence emission was collected using a $60 \times 1.00 \mathrm{NA}$ water dipping objective from the top perpendicular to the illumination sheet. Cells were alternately excited at 405 and $488 \mathrm{~nm}$, and emission was collected at $513 \mathrm{~nm}$. Chondrocytes were imaged for $1 \mathrm{~min}$ before the addition of $25 \mu \mathrm{M}$ menadione, $25 \mu \mathrm{M}$ DMNQ, or DMSO vehicle control. Images were taken every $10 \mathrm{~s}$ for a total of 2 min and analyzed using FIJI Image J 1.51t (22) using techniques based on the sensitized FRET method as previously reported (18). Briefly, individual cells were segmented using standard thresholding techniques and $\mathrm{H}_{2} \mathrm{O}_{2}$ levels were determined ratiometrically on a pixel-by-pixel basis using the following equation at each time point:

$$
I=\frac{405 e x}{488 e x}
$$

where $I$ corresponds to $\mathrm{H}_{2} \mathrm{O}_{2}$ levels, and $405 \mathrm{ex}$ and $488 \mathrm{ex}$ correspond to the observed intensity of each respective image channel.

\subsection{Analysis of Prx redox status}

Cells were cultured overnight in serum-free conditions and then treated with $25 \mu \mathrm{M}$ menadione or $25 \mu \mathrm{M}$ DMNQ. Reduced, oxidized and hyperoxidized forms of Prxs were analysed as previously described $(18,23)$. Briefly, cultured cells were treated with menadione or DMNQ for the indicated times, washed in 1X PBS and incubated in an NEMcontaining alkylating buffer (40 mM HEPES, $50 \mathrm{mM} \mathrm{NaCl}, 1 \mathrm{mM}$ EGTA, 200 units $/ \mathrm{mL}$ catalase, $100 \mathrm{mM}$ NEM, PMSF and phosphatase inhibitor cocktail 2 (pH7.4)) for $10 \mathrm{~min}$ prior to lysis in order to alkylate reduced thiols and block artificial oxidation that may arise due to lysis. NEM alkylating buffer was discarded and cells were lysed at $4^{\circ} \mathrm{C}$ under gentle agitation for $30 \mathrm{~min}$ in a standard lysis buffer containing 200 units $/ \mathrm{mL}$ catalase and $100 \mathrm{mM}$ NEM, PMSF and phosphatase inhibitor cocktail 2 (pH 7.4). To remove the insoluble protein fraction, cell lysates were centrifuged at 13,000 rpm for $10 \mathrm{~min}$. Soluble protein concentrations were quantified using the Pierce Micro BCA kit (Thermo Scientific). Protein lysates were boiled and immunoblotted under reducing or non-reducing conditions (in the presence or absence of $10 \% \beta$-mercaptoethanol) as described (24). For identification of global Prx hyperoxidation, cell lysates were immunoblotted under reducing conditions and 
probed with an antibody that reacts with Prx 1-Prx4 when in the Prx-SO $\mathrm{SO}_{2 / 3}$ state (18). $\beta$-actin or $\beta$-tubulin were used as loading controls.

\subsection{Analysis of chondrocyte intracellular signaling}

Overnight incubation of chondrocytes in serum-free containing media preceded experimental treatments. Chondrocytes were treated with $25 \mu \mathrm{M}$ menadione, $25 \mu \mathrm{M}$ DMNQ, $50 \mathrm{ng} / \mathrm{mL}$ IGF-1 or pretreated with menadione or DMNQ for $30 \mathrm{~min}$ prior to stimulation with IGF-1 for the indicated time points. All cell signaling immunoblots were performed under reducing conditions using phospho-specific antibodies, with antibodies to total protein serving as loading controls, with the exception of phospho c-Jun, which was normalized to $\beta$-actin. The effects of menadione and DMNQ on cell viability were measured using the LIVE/DEAD cell assay kit (Molecular Probes) (25).

\subsection{Statistical analysis}

All densitometric analysis of immunoblots was performed using ImageJ software. Data analysis was conducted in GraphPad Prism version 7. All data are presented as mean values \pm SEM. In all cases, independent experiments were performed using cells cultured from different tissues donors. Exact biological replicates (from independent donors) are indicated in figure legends. Results were analysed by $t$-test when comparing two groups or one or twoway ANOVA for comparison of more than two groups. Post-hoc corrections were applied as appropriate and a $p$-value of $<0.05$ was deemed significant.

\subsection{Study Approval}

Use of human tissue was in accordance with the Institutional Review Board at the University of North Carolina at Chapel Hill and Rush University Medical Center.

\section{Results}

\subsection{Menadione and DMNQ generate similar levels of cytosolic $\mathrm{H}_{2} \mathrm{O}_{2}$, but originating from different cellular compartments.}

As measured by the Orp1-roGFP based $\mathrm{H}_{2} \mathrm{O}_{2}$ biosensor, treatment of articular chondrocytes with menadione or DMNQ led to comparable levels of intracellular $\mathrm{H}_{2} \mathrm{O}_{2}$ generation within $20 \mathrm{~s}$ (Figure 1A, B). To assess mitochondrial levels of $\mathrm{H}_{2} \mathrm{O}_{2}$ generated in response to menadione and DMNQ, $\mathrm{H}_{2} \mathrm{O}_{2}$ levels were measured using a mitochondrially targeted $\mathrm{H}_{2} \mathrm{O}_{2}$ redox biosensor (Mito-Orp1-roGFP). Treatment with menadione led to an increase in mitochondrial $\mathrm{H}_{2} \mathrm{O}_{2}$ generation compared to controls. Treatment with DMNQ generated relatively lower levels of mitochondrial $\mathrm{H}_{2} \mathrm{O}_{2}$ when compared to menadione-induced $\mathrm{H}_{2} \mathrm{O}_{2}$ generation (Fig 1C, D). These data taken together suggest that menadione generates $\mathrm{H}_{2} \mathrm{O}_{2}$, at least partially, in the mitochondria whereas DMNQ generates $\mathrm{H}_{2} \mathrm{O}_{2}$ primarily extramitochondrially.

\subsection{Menadione and DMNQ induce differential Prx hyperoxidation in chondrocytes.}

To assess the ability of menadione and DMNQ-generated $\mathrm{H}_{2} \mathrm{O}_{2}$ to hyperoxidize the Prxs and induce intracellular oxidative stress in human chondrocytes, immunoblotting was performed 
using an antibody that specifically detects the hyperoxidized $\left(\operatorname{PrxSO}_{2 / 3}\right)$ forms of Prx1-Prx4. We have previously identified the precise molecular weights of hyperoxidized Prx1-Prx3 in chondrocytes when we immunoblotted under reducing conditions with the antibody to $\operatorname{PrxSO}_{2 / 3}$ while Prx4 was more difficult to identify (18) and so we have focused the current work on Prx1-Prx3. Both menadione and DMNQ led to a time-dependent increase in hyperoxidation of Prx1-Prx3 compared to untreated controls (Figure 2). No difference in total Prx hyperoxidation was observed between the two treatments over the time course studied (Figure 2A,B), a finding that is consistent with the Orp1-roGFP data.

To further analyze the oxidation of specific Prxs, human chondrocytes were treated with either menadione or DMNQ and Prx isoforms were analysed by immunoblotting under nonreducing conditions. This confirmed our previously published data (18) where we demonstrated Prx 2 as both an oxidized dimer migrating at $44 \mathrm{kDa}$ and a reduced monomeric form that migrated at $21 \mathrm{kDa}$. Within $5 \mathrm{~min}$ of menadione or DMNQ treatment, the reduced monomeric form of Prx 2 disappeared in a way that was proportional to the appearance of the dimer, thus indicating Prx 2 oxidation (Figure 2C). The intensity of the oxidized dimer band decreased over the $60 \mathrm{~min}$ time course, accompanied by the appearance of the band corresponding to the hyperoxidized, monomeric form (Figure 2C). No significant differences in Prx2 hyperoxidized monomer formation were observed between menadione and DMNQ (Figure 2C, F).

As we have previously reported (18), under non-reducing conditions, Prx3 was present in the monomeric form at approximately $24 \mathrm{kDa}$ and the oxidized, dimeric form was present at approximately $38 \mathrm{kDa}$ (Figure 2D). Similar to Prx2, menadione and DMNQ treatment led to increased formation of the oxidized, disulfide form of Prx 3 within 5 min. However, menadione treatment caused hyperoxidation of Prx3 at 30 and $60 \mathrm{~min}$ (Figure 2D) but DMNQ treatment, in contrast, did not cause Prx3 hyperoxidation. Indeed, a significant difference was observed for hyperoxidized Prx3 monomer formation between menadione and DMNQ treatments at 60 min (Figure 2F).

We next examined the effect of menadione and DMNQ on the redox status of Prx 1. Monomeric Prx 1 was observed at approximately $22 \mathrm{kDa}$ and oxidized, dimeric Prx 1 was observed at $38 \mathrm{kDa}$ (Figure 2E). Under unstimulated conditions, Prx 1 was observed primarily in the monomeric form with low levels of oxidized dimer. Treatment with menadione and DMNQ led to oxidation and high levels of Prx1 dimer formation within 5 min (Figure 2E). Treatment with menadione did not cause hyperoxidation of Prx1, as evidenced by maintenance of the oxidized dimer and the absence of hyperoxidized monomer formation at $60 \mathrm{~min}$. DMNQ treatment, however, led to a time-dependent decrease in oxidized dimer formation between 5-60 min and a consistently observed increase in the hyperoxidized monomer at 60 min indicating DMNQ-induced Prx 1 hyperoxidation (Figure 2E). DMNQ treatment induced significantly higher levels of Prx1 hyperoxidation than menadione treatment (Figure 2F). In order to explore Prx1 regulation further, chondrocyte lysates were immunoblotted with an antibody to phosphorylated Prx1 (Tyr194), a phosphorylation site that has been previously reported to inhibit Prx1 peroxidase activity (12). Treatment with menadione, but not DMNQ, led to a time-dependent increase in Prx1 phosphorylation, which was maximal at 60 min (Figure 2G). 
These findings altogether led to the conclusion that mainly through their preferential generation of $\mathrm{H}_{2} \mathrm{O}_{2}$ in different cellular compartments, menadione and DMNQ differentially regulate Prx1-Prx3 hyperoxidation as well as phosphorylation of Prx1, providing for an opportunity to determine the effects on cell signaling when different Prxs are inhibited under conditions of elevated $\mathrm{H}_{2} \mathrm{O}_{2}$.

\subsection{Differential Prx oxidation in response to menadione and DMNQ is associated with distinctly different effects on IGF-1 and MAP kinase signaling pathways.}

In order to examine the role of Prx hyperoxidation on downstream signaling events, we chose to assess activation of specific cell signaling pathways pertinent to anabolic and catabolic processes within the articular joint that are implicated in the joint disease osteoarthritis (OA). We focused on redox regulation of the chondroprotective and proanabolic insulin-like growth factor-1 (IGF-1) signaling pathway that we have shown is inhibited under conditions of oxidative stress $(18,26)$. For comparison, we examined the procatabolic $\mathrm{p} 38$, extracellular-signal regulated kinase (ERK) and c-Jun $\mathrm{N}$-terminal kinase (JNK) pathways that are largely implicated in chondrocyte cell death and/or cartilage extracellular matrix degradation (18,27-30). Primary human chondrocytes were treated with menadione, DMNQ, or IGF-1 for $60 \mathrm{~min}$, or pre-treated with menadione or DMNQ for 30 min prior to addition of IGF-1 for $60 \mathrm{~min}$, and phosphorylation of proteins pertinent to the IGF-1 and MAP kinase signaling pathways were determined. Treatment of human chondrocytes with IGF-1 stimulated phosphorylation of the IGF-1 receptor and the downstream kinase Akt (Figure 3A, B, C). Phosphorylation of proline rich Akt substrate 40 (PRAS40) was analysed as a marker of Akt kinase activity and was significantly increased with IGF-1 treatment (Figure 3A, D). IGF-1 treatment did not lead to increased phosphorylation of p38, ERK, JNK, or c-Jun (Figure 3A-H). Under conditions shown above where we observed Prx hyperoxidation in response to menadione or DMNQ, we did not detect significant phosphorylation of the IGF-1 receptor, Akt or PRAS40. However, menadione significantly increased levels of phosphorylated p38 (Figure 3A-E) but not ERK (Figure 3A, F). Prx hyperoxidation in response to menadione was associated with weak JNK phosphorylation but not with increased phosphorylation of the JNK downstream substrate and marker of activity, c-Jun (Figure 3A, G, H). Similar to menadione, DMNQ also increased levels of phosphorylated p38, but not ERK. However, in contrast to menadione, DMNQ-induced Prx 1 and Prx 2 hyperoxidation was associated with high levels of JNK phosphorylation. This correlated with strong phosphorylation of all serine and threonine sites of c-Jun as indicated by an electrophoretic shift of the c-Jun monomer (Figure 3A, G, H).

Previous studies from our group $(18,29)$ have demonstrated that oxidative stress inhibits IGF-1induced phosphorylation of pro-survival Akt signaling in chondrocytes. To compare the effect of menadione and DMNQ on IGF signaling, we pre-treated human chondrocytes with these compounds for 30 min to induce oxidative stress and then treated with IGF-1 for the indicated times (0-60 min) (Figure 4). The Prx hyperoxidation in response to pretreatment with menadione or DMNQ noted above had no effect on the ability of IGF-1 to stimulate IGF-receptor phosphorylation over $30 \mathrm{~min}$ while at $60 \mathrm{~min}$, menadione slightly increased IGF-1 receptor phosphorylation (Figure 4A, B). Consistent with our previous 
report (18), Prx hyperoxidation induced by menadione was associated with significant inhibition of IGF-1 stimulated Akt phosphorylation, which in turn, also decreased phosphorylation of its downstream substrate and marker of activation, PRAS40 (Figure 4A,C,D). In contrast, DMNQ-induced Prx hyperoxidation had no effect on Akt or PRAS40 phosphorylation in the presence of IGF-1. As shown in Figure 3, treatment with menadione led to phosphorylation of $\mathrm{p} 38$, whereas DMNQ treatment led to phosphorylation of $\mathrm{p} 38$, JNK and c-Jun. The addition of IGF-1 did not alter this pattern (Figure 4A, E-H).

\subsection{Prx hyperoxidation in response to menadione and DMNQ is associated with cell death in human chondrocytes.}

As menadione and DMNQ exerted differential effects on Prx hyperoxidation and on prosurvival and pro-catabolic signal transduction, we aimed to test the effects on chondrocyte cell viability. Prx hyperoxidation in response to menadione was associated with reduced cell viability to $73.2 \pm 5.2 \%$ by $3 \mathrm{~h}$ and led to complete cell death within $6 \mathrm{~h}$ (Figure $4 \mathrm{I}$ ). In contrast, when compared to control, DMNQ-induced Prx hyperoxidation did not significantly alter cell viability over $6 \mathrm{~h}$, but reduced cell viability to $53 \pm 10.2 \%$ at $9 \mathrm{~h}$ with complete cell death observed at $18 \mathrm{~h}$. Significant differences between menadione and DMNQ treatments were observed at 3, 6 and $9 \mathrm{~h}$ respectively (Figure $4 \mathrm{I}$ ).

\subsection{Overexpression of Prx3 but not Prx1 or Prx2 decreases Prx hyperoxidation}

In order to assess if adenoviral overexpression of specific Prxs could alter oxidative stressinduced Prx hyperoxidation, chondrocytes were transduced with adenoviral vectors encoding cytosolic Prx1 (ad-Prx1) or Prx2 (ad-Prx2), or mitochondrial Prx3 (ad-Prx3). Immunoblots for Prx1-Prx3 in cells overexpressing the Prxs were compared to null empty vector controls (ad-Null) to confirm Prx protein overexpression (Figure 5A). Cells were then treated with menadione or DMNQ for 0-60 min and immunoblotting for $\mathrm{PrxSO}_{2 / 3}$ to detect Prx hyperoxidation levels was performed. As expected, in chondrocytes transduced with the null empty vector control, treatment with menadione and DMNQ led to a time-dependent increase in $\mathrm{PrxSO}_{2 / 3}$ formation (Figure 5B-G). No significant differences in total $\mathrm{PrxSO}_{2 / 3}$ formation were observed with either menadione or DMNQ treatment when chondrocytes were transduced with adenoviral vectors encoding Prx 1 or Prx2, compared to null empty vector control (Figure 5B-G). However, when cells were transduced with ad-Prx2 and treated with menadione or DMNQ, we observed preferential hyperoxidation of Prx2, as evidenced by a marked increase in band intensity corresponding to $\operatorname{Prx} 2 \mathrm{SO}_{2 / 3}$, and a slight decrease in bands corresponding to $\operatorname{Prx} 1 \mathrm{SO}_{2 / 3}$ and $\operatorname{Prx} 3 \mathrm{SO}_{2 / 3}$ (Figure 5D, F). Importantly, overexpression of mitochondrial Prx3 significantly decreased menadione-induced $\operatorname{PrxSO}_{2 / 3}$ formation when compared to null empty vector controls at 30 and 60 min (Figure 5D,E) but had no significant effect on DMNQ-induced $\mathrm{PrxSO}_{2 / 3}$ formation (Figure 5F,G). This would be consistent with menadione initially generating $\mathrm{H}_{2} \mathrm{O}_{2}$ in the mitochondria where $\operatorname{Prx} 3$ is located while DMNQ generates $\mathrm{H}_{2} \mathrm{O}_{2}$ primarily in the cytosol.

\subsection{Overexpression of Prx3 but not Prx1 or Prx2 attenuates menadione-induced Akt inhibition and $\mathrm{p} 38$ phosphorylation.}

In order to determine if attenuation of Prx hyperoxidation by Prx overexpression could alter MAP kinase signaling or restore signaling responses to IGF-1, we transduced human 
chondrocytes with adenoviral vectors encoding Prx1, Prx2, Prx3 or a null empty vector control and analyzed phosphorylation levels of proteins pertinent to IGF-1 and MAP kinase signaling pathways. Interestingly, overexpression of Prx 1 led to enhanced basal Akt and PRAS40 phosphorylation levels that were significantly higher than basal levels in cells transduced with the null empty vector control (Figure 6A-C). However, overexpression of Prx 1 had no effect on menadione-induced MAP kinase or IGF-1 cell signaling events when compared to control treated cells (Figure 6A-E). Similar to the findings with menadione, Prx 1 overexpression also had no effect on DMNQ-induced MAP kinase or IGF-1 cell signaling events when compared to null empty vector treated cells (Figure 6F-J). Despite a consistent and unexpected trend towards increased DMNQ-induced MAP kinase signaling when cells were transduced with the Prx 1 adenoviral vector, significant differences between Prx1 overexpressing cells and null vector treated cells were not observed (Figure 6F-J).

Unlike Prx1, overexpression of Prx2 did not significantly alter basal Akt or PRAS40 phosphorylation (Figure 7A-C). Similar to Prx1, Prx2 overexpression did not alter any phosphorylation events induced by menadione (Figure 7A-E). Although overexpression of Prx2 trended towards decreasing DMNQ-induced JNK and c-Jun phosphorylation, this effect was not significant at any time point when compared to null vector treated cells (Figure 7F-H). Prx2 overexpression did not alter phosphorylation of p38 but partially decreased ERK phosphorylation in the presence of IGF-1 when compared to the null control (Figure 7F, I, J).

Importantly, overexpression of Prx3 abrogated menadione-induced inhibition of IGF-1stimulated Akt and PRAS40 phosphorylation (Figure 8A-C). Overexpression of Prx3 also significantly reduced phosphorylation of $\mathrm{p} 38$ and reduced ERK phosphorylation in the presence of IGF-1 when compared to empty vector control conditions (Figure 8A, D, E). The effects of overexpression of Prx 3 to modify DMNQ mediated cell signaling events were also tested. Prx3 overexpression had no effects on DMNQ mediated cell signaling (Figure $8 \mathrm{~F}-\mathrm{J})$, an effect which is consistent with our data indicating that DMNQ primarily induces cytosolic $\mathrm{H}_{2} \mathrm{O}_{2}$.

\section{Discussion}

Recent advances in redox biology implicate Prx oxidation as a key mechanism for regulating intracellular $\mathrm{H}_{2} \mathrm{O}_{2}$ levels and subsequent downstream cell signaling events. As such, this study chose to explore the effects of $\mathrm{H}_{2} \mathrm{O}_{2}$-induced Prx1-Prx3 oxidation, as well as Prx 1 phosphorylation, on selected downstream cell signaling events using human articular chondrocytes as a cell model system. We found that menadione and DMNQ could be used as $\mathrm{H}_{2} \mathrm{O}_{2}$-producing tools that resulted in differential hyperoxidation profiles of $\operatorname{Prx} 1-\operatorname{Prx} 3$ as well as phosphorylation of Prx1 that were associated with changes in specific redoxregulated signaling pathways. Menadione induced hyperoxidation of Prx 2 and $\operatorname{Prx} 3$ but not Prx 1, consistent with our previous findings (18), but also induced phosphorylation of Prx 1 at Tyr194 that, like hyperoxidation, would be expected to inhibit Prx1 activity (12). In contrast, DMNQ induced hyperoxidation of Prx1 and Prx2. Although DMNQ led to oxidation of Prx3, we found no evidence that DMNQ treatment led to Prx3 hyperoxidation. Because Prx3 is located in the mitochondria, these findings suggested menadione generated higher levels 
of mitochondrial $\mathrm{H}_{2} \mathrm{O}_{2}$ than DMNQ, which was supported by the findings using the mitochondrial-targeted Orp-1-roGFP. The menadione-induced hyperoxidation of Prx 2 and Prx3 and phosphorylation of Prx 1 was associated with increased phosphorylation of p38 and inhibition of IGF-1-induced Akt activity while DMNQ-induced hyperoxidation of Prx 1 and Prx2 was associated with phosphorylation of p38 and JNK activation without inhibition of IGF-1 stimulated Akt activity. These findings suggested that Prx3 hyperoxidation in the presence of menadione was responsible for the loss of Akt activity in response to IGF-1. This was supported by the finding that overexpression of Prx3, but not Prx1 or Prx2, inhibited menadione-induced Prx3 hyperoxidation and restored the Akt response to IGF-1.

Unlike the inhibitory effects on IGF-1 activation of Akt, both menadione and DMNQ stimulated phosphorylation of p38. However, similar to the Akt studies, overexpression of mitochondrial Prx3 that was sufficient to reduce Prx1-Prx3 hyperoxidation, but not overexpression of Prx 1 or Prx2, was able to block the ability of menadione but not DMNQ to stimulate $\mathrm{p} 38$ phosphorylation. This suggests that mitochondrial $\mathrm{H}_{2} \mathrm{O}_{2}$ was the source for phosphorylation of p38 in response to menadione. The finding that hyperoxidation of $\operatorname{Prx} 3$ is associated with enhanced p38 phosphorylation agrees with the work of Kil et al (31) who demonstrated that peritoneal injection of adrenocorticotropic hormone leads to increased mitochondrial $\mathrm{H}_{2} \mathrm{O}_{2}$ generation and subsequent Prx3 hyperoxidation and $\mathrm{p} 38$ activation in the adrenal glands of mice. The ability of overexpression of mitochondrial Prx 3 to inhibit hyperoxidation of the cytosolic Prx 1 and $\operatorname{Prx} 2$ in chondrocytes is consistent with studies demonstrating that knockdown of $\operatorname{Pr} 33$ decreases scavenging of $\mathrm{H}_{2} \mathrm{O}_{2}$ in both mitochondrial and cytosolic compartments in cardiac myoblasts (32).

Although both menadione and DMNQ induced p38 phosphorylation, only DMNQ induced significant JNK phosphorylation that was associated with phosphorylation of the JNK substrate c-Jun, indicating that the phosphorylated JNK was also active. This was important to determine since we have recently shown that when the JNK isoform JNK2 is oxidized, its kinase activity is decreased independent of its phosphorylation status (33). In that study, like the current study, we found menadione stimulated p38 but not JNK phosphorylation and in addition discovered menadione inhibited JNK phosphorylation (but not p38 phosphorylation) stimulated by fibronectin fragments that signal through the a $5 \beta 1$ integrin. Compared to menadione, fibronectin fragments generate very low levels of $\mathrm{H}_{2} \mathrm{O}_{2}$ and so we had hypothesized that the high levels of $\mathrm{H}_{2} \mathrm{O}_{2}$ generated by menadione were inhibiting JNK (18). However, in the present study DMNQ did not inhibit JNK activation despite generating similar levels of cytosolic $\mathrm{H}_{2} \mathrm{O}_{2}$ as menadione suggesting that perhaps mitochondrial $\mathrm{H}_{2} \mathrm{O}_{2}$ and Prx3 inactivation induced by menadione were responsible for JNK inhibition. The differential menadione and DMNQ activation of $\mathrm{p} 38$ and JNK, both of which are activated by upstream, redox sensitive ASK1 phosphorylation (34), demonstrates that these signaling cascades can be independently regulated in response to specific levels and types of oxidative stimuli, suggestive of a high degree of specificity for a finely tuned redox response.

We hypothesised that targeting the Prxs, in order to reduce oxidative stress levels, may restore homeostatic cell signaling events in chondrocytes through maintenance of redox balance and abrogation of oxidative stress-induced catabolic cell signaling. To this end, we tested the effect of overexpression of Prx 1, 2, and 3 to alter menadione and DMNQ-induced 
MAP kinase and Akt signaling events. Recent data suggest that overexpression of Prx 1 decreases IL- $1 \beta$-induced ROS production and apoptosis in OA chondrocytes (35) and can supress JNK activity in lung cancer cells (36). These findings are in contrast with those in the present study, in which we did not observe an effect of Prx 1 overexpression on oxidative stress-induced Prx hyperoxidation or downstream MAP kinase signaling events. We did, however, observe an increase in basal levels of Akt activity in cells overexpressing Prx1, which indicates that Prx 1 may promote pro-anabolic cell signaling events at least to some extent, in chondrocytes. Similar to the findings for Prx 1, overexpression of Prx 2 did not alter DMNQ or menadione-induced MAP kinase signaling and had no effect on Akt signaling. Notably, when assessing Prx hyperoxidation using the antibody to $\operatorname{PrxSO}_{2 / 3}$ we consistently observed that Prx2, when overexpressed, was preferentially hyperoxidized by both menadione and DMNQ. This is likely due to the enhanced sensitivity of Prx2 to hyperoxidation (16,17). Preferential hyperoxidation of overexpressed Prx2 would inhibit its function and therefore not contribute to attenuating catabolic signaling induced by oxidative stress levels of $\mathrm{H}_{2} \mathrm{O}_{2}$.

Unlike Prx2, Prx3, which is more resistant to hyperoxidation than cytosolic Prxs (15-17), was not hyperoxidized when overexpressed and thus, was able to contribute to redox homeostasis and subsequent regulation of cytosolic cell signaling pathways. The importance of maintaining Prx3 activity has been highlighted by the work of Noh and colleagues (37) who demonstrated that in response to oxidative stress, overexpression of mitochondrial sulfiredoxin enhanced Prx 3 regeneration from the hyperoxidized, inactive form, to the reduced, catalytically active form. This increased Prx3 regeneration was associated with decreased apoptosis and preservation of mitochondrial membrane potentials (37).

The current study utilized chondrocytes isolated from human articular cartilage in order to investigate the role of oxidative stress in $\mathrm{OA}$ which is characterized by excessive cartilage destruction. Aging represents a key OA risk factor and the aging OA joint presents with enhanced levels of oxidative stress markers and mitochondrial dysfunction $(18,38-41)$. Although the specific mechanisms linking oxidative stress to OA are yet to be fully elucidated, recent data suggest that an age-related increase in oxidative stress contributes to OA development and progression by disturbing chondrocyte anabolic and catabolic signaling events to promote cartilage matrix degradation and induce cell death $(18,26,27,29,30)$. Mitochondrial dysfunction is regarded as a 'hallmark of aging' (42). As the mitochondria are a key source of ROS, an age-related decline in mitochondrial function has been proposed to cause increases in oxidative stress-induced cellular damage, ultimately promoting age-related pathogenesis (5). As such, targeting the mitochondria to restore redox balance has the potential to be beneficial in OA. Data from the present study are in accordance with our previous work which reported that catalase targeted to the mitochondria (MCAT) attenuates menadione-induced Prx3 hyperoxidation and activation of $\mathrm{p} 38$ to maintain chondrocyte cell viability (18). Transgenic MCAT mice were also protected from age-related OA when compared to wild type control mice. This effect was attributed to reduced oxidative stress levels, as measured by a reduction in Prx hyperoxidation, and reduced p38 signaling (18). 
In chondrocytes, Prx 1 appears to be regulated by both phosphorylation and hyperoxidation in a manner that is stimulus dependent. Prxs, when hyperoxidized, can be regenerated and returned to the Prx catalytic cycle by the enzyme sulfiredoxin but this is a slow process which also requires ATP (9). It is conceivable, therefore, that phosphorylation of Prx1, which can be potentially reversed by phosphatases, could constitute a mechanism whereby Prx 1 can be more transiently inhibited relative to the inhibition that occurs with hyperoxidation. It was interesting that menadione, but not DMNQ, induced Prx1 phosphorylation while DMNQ, but not menadione, induced Prx1 hyperoxidation. We do not have a ready explanation for this difference but it is possible that either Prx 1 hyperoxidation inhibits phosphorylation or vice-versa, phosphorylation may inhibit Prx 1 hyperoxidation (which would in fact be expected given inhibited Prx1 catalytic turnover).

In conclusion, the findings from the current study suggest that targeted therapeutic approaches aimed at maintaining mitochondrial Prx3 activity may represent a novel strategy to restore homeostatic cell signaling events in chondrocytes under conditions of mitochondrial-mediated oxidative stress. This may be particularly useful in age-related diseases of the joint such as OA where enhanced mitochondrial dysfunction and oxidative stress are proposed to disturb normal cell signaling events to promote cellular and matrix damage (43).

\section{Acknowledgements:}

We thank the Gift of Hope Organ and Tissue Donor Network and the donor families for providing human donor tissue. We thank Dr. Arcady Margulis for tissue procurement and Mrs. Arnavaz Hakimiyan from Rush University Medical Center for technical assistance. We thank Brian O'Rourke for the mito-Orp-1 roGFP redox biosensor. We thank Kathryn Kelley and Mary Zhou for technical assistance and Robert Currin and the University of North Carolina School of Medicine Hooker Imaging Core for access and assistance with confocal microscopy. We also thank Fangjia Li for assistance with oSPIM microscopy.

Funding sources: This project was supported by a grant to RFL from the National Institute on Aging (AG044034), to LBP from the National Institute of General Medicine (GM119227) and to JAC from the American Federation for Aging Research. STW was supported by a grant from the National Science Foundation/EPSCoR Cooperative Agreement \#IIA-1355423 and by the State of South Dakota. This study was also supported by the Klaus Kuettner Chair for Osteoarthritis Research (SC), Rush University Medical Center. Any opinions, findings, and conclusions or recommendations expressed in this material are those of the authors and do not necessarily reflect the views of the National Institutes on Aging or the National Science Foundation.

\section{Abbreviations}

DMNQ

ERK

IGF-1

JNK

menadione

MCAT

NEM

\section{2,3-dimethyl-1,4-naphthoquinone}

extracellular-signal regulated kinase

insulinlike growth factor 1

c-Jun N-terminal kinase

2-methyl-1,4-naphthoquinone

catalase targeted to the mitochondria

N-ethylmaleimide 


$\begin{array}{ll}\text { PRAS40 } & \text { proline rich Akt substrate } \\ \text { Prx } & \text { peroxiredoxin } \\ \text { Prx-SOH } & \text { peroxiredoxin-sulfenic acid } \\ \text { Prx-SO }_{2} \mathrm{H} & \text { peroxiredoxin-sulfinic acid } \\ \text { Prx-SO }_{3} \mathrm{H} & \text { peroxiredoxin-sulfonic acid } \\ \text { ROS } & \text { reactive oxygen species }\end{array}$

\section{References}

1. Schieber M, and Chandel NS (2014) ROS function in redox signaling and oxidative stress. Curr Biol 24, R453-462 [PubMed: 24845678]

2. Finkel T (2011) Signal transduction by reactive oxygen species. J Cell Biol 194, 7-15 [PubMed: 21746850]

3. Sies H (2017) Hydrogen peroxide as a central redox signaling molecule in physiological oxidative stress: Oxidative eustress. Redox Biol 11, 613-619 [PubMed: 28110218]

4. Klomsiri C, Karplus PA, and Poole LB (2011) Cysteine-based redox switches in enzymes. Antioxid Redox Signal 14, 1065-1077 [PubMed: 20799881]

5. Jones DP (2015) Redox theory of aging. Redox Biol 5, 71-79 [PubMed: 25863726]

6. Adimora NJ, Jones DP, and Kemp ML (2010) A model of redox kinetics implicates the thiol proteome in cellular hydrogen peroxide responses. Antioxid Redox Signal 13, 731-743 [PubMed: 20121341]

7. Wood ZA, Poole LB, and Karplus PA (2003) Peroxiredoxin evolution and the regulation of hydrogen peroxide signaling. Science 300, 650-653 [PubMed: 12714747]

8. Perkins A, Nelson KJ, Parsonage D, Poole LB, and Karplus PA (2015) Peroxiredoxins: guardians against oxidative stress and modulators of peroxide signaling. Trends Biochem Sci 40, 435-445 [PubMed: 26067716]

9. Lowther WT, and Haynes AC (2011) Reduction of cysteine sulfinic acid in eukaryotic, typical 2-Cys peroxiredoxins by sulfiredoxin. Antioxid Redox Signal 15, 99-109 [PubMed: 20712415]

10. Randall LM, Ferrer-Sueta G, and Denicola A (2013) Peroxiredoxins as preferential targets in H2O2-induced signaling. Methods Enzymol 527, 41-63 [PubMed: 23830625]

11. Veal EA, Underwood ZE, Tomalin LE, Morgan BA, and Pillay CS (2018) Hyperoxidation of peroxiredoxins: Gain or loss of function? Antioxid Redox Signal 28, 574-590 [PubMed: 28762774]

12. Woo HA, Yim SH, Shin DH, Kang D, Yu DY, and Rhee SG (2010) Inactivation of peroxiredoxin I by phosphorylation allows localized $\mathrm{H} 2 \mathrm{O} 2$ accumulation for cell signaling. Cell 140, 517-528 [PubMed: 20178744]

13. Sobotta MC, Liou W, Stocker S, Talwar D, Oehler M, Ruppert T, Scharf AN, and Dick TP (2015) Peroxiredoxin-2 and STAT3 form a redox relay for $\mathrm{H} 2 \mathrm{O} 2$ signaling. Nat Chem Biol 11, 64-70 [PubMed: 25402766]

14. Rhee SG, Woo HA, Kil IS, and Bae SH (2012) Peroxiredoxin functions as a peroxidase and a regulator and sensor of local peroxides. J Biol Chem 287, 4403-4410 [PubMed: 22147704]

15. Cox AG, Pearson AG, Pullar JM, Jonsson TJ, Lowther WT, Winterbourn CC, and Hampton MB (2009) Mitochondrial peroxiredoxin 3 is more resilient to hyperoxidation than cytoplasmic peroxiredoxins. Biochem J 421, 51-58 [PubMed: 19356151]

16. Peskin AV, Dickerhof N, Poynton RA, Paton LN, Pace PE, Hampton MB, and Winterbourn CC (2013) Hyperoxidation of peroxiredoxins 2 and 3: rate constants for the reactions of the sulfenic acid of the peroxidatic cysteine. J Biol Chem 288, 14170-14177 [PubMed: 23543738] 
17. Bolduc JA, Nelson KJ, Haynes AC, Lee J, Reisz JA, Graff AH, Clodfelter JE, Parsonage D, Poole LB, Furdui CM, and Lowther WT (2018) Novel hyperoxidation resistance motifs in 2-Cys peroxiredoxins. J Biol Chem 293, 11901-11912 [PubMed: 29884768]

18. Collins JA, Wood ST, Nelson KJ, Rowe MA, Carlson CS, Chubinskaya S, Poole LB, Furdui CM, and Loeser RF (2016) Oxidative stress promotes peroxiredoxin hyperoxidation and attenuates prosurvival signaling in aging chondrocytes. J Biol Chem 291, 6641-6654 [PubMed: 26797130]

19. Loeser RF, Pacione CA, and Chubinskaya S (2003) The combination of insulin-like growth factor 1 and osteogenic protein 1 promotes increased survival of and matrix synthesis by normal and osteoarthritic human articular chondrocytes. Arthritis Rheum 48, 2188-2196 [PubMed: 12905472]

20. Watanabe N, and Forman HJ (2003) Autoxidation of extracellular hydroquinones is a causative event for the cytotoxicity of menadione and DMNQ in A549-S cells. Arch Biochem Biophys 411, 145-157 [PubMed: 12590933]

21. Vilela M, Halidi N, Besson S, Elliott H, Hahn K, Tytell J, and Danuser G (2013) Fluctuation Analysis of Activity Biosensor Images for the Study of Information Flow in Signaling Pathways in Fluorescence Fluctuation Spectroscopy (Tetin SY ed.), Elsevier Academic Press Inc, San Diego pp 253-276

22. Schneider CA, Rasband WS, and Eliceiri KW (2012) NIH Image to ImageJ: 25 years of image analysis. Nat Methods 9, 671-675 [PubMed: 22930834]

23. Cox AG, Winterbourn CC, and Hampton MB (2010) Measuring the redox state of cellular peroxiredoxins by immunoblotting. Methods Enzymol 474, 51-66 [PubMed: 20609904]

24. Del Carlo M Jr., and Loeser RF (2002) Nitric oxide-mediated chondrocyte cell death requires the generation of additional reactive oxygen species. Arthritis Rheum 46, 394-403 [PubMed: 11840442]

25. Del Carlo B, Pellegrini M, and Pellegrino M (2002) Calmodulin antagonists do not inhibit IK(Ca) channels of human erythrocytes. Biochim Biophys Acta 1558, 133-141 [PubMed: 11779563]

26. Yin W, Park JI, and Loeser RF (2009) Oxidative stress inhibits insulin-like growth factor-I induction of chondrocyte proteoglycan synthesis through differential regulation of phosphatidylinositol 3-Kinase-Akt and MEK-ERK MAPK signaling pathways. J Biol Chem 284, 31972-31981 [PubMed: 19762915]

27. Forsyth CB, Cole A, Murphy G, Bienias JL, Im HJ, and Loeser RF Jr. (2005) Increased matrix metalloproteinase-13 production with aging by human articular chondrocytes in response to catabolic stimuli. J Gerontol A Biol Sci Med Sci 60, 1118-1124 [PubMed: 16183949]

28. Greene MA, and Loeser RF (2015) Function of the chondrocyte PI-3 kinase-Akt signaling pathway is stimulus dependent. Osteoarthr Cartilage 23, 949-956

29. Loeser RF, Gandhi U, Long DL, Yin W, and Chubinskaya S (2014) Aging and oxidative stress reduce the response of human articular chondrocytes to insulin-like growth factor 1 and osteogenic protein 1. Arthritis Rheumatol 66, 2201-2209 [PubMed: 24664641]

30. Wood ST, Long DL, Reisz JA, Yammani RR, Burke EA, Klomsiri C, Poole LB, Furdui CM, and Loeser RF (2016) Cysteine-mediated redox regulation of cell signaling in chondrocytes stimulated with fibronectin fragments. Arthritis Rheumatol 68, 117-126 [PubMed: 26314228]

31. Kil IS, Lee SK, Ryu KW, Woo HA, Hu MC, Bae SH, and Rhee SG (2012) Feedback control of adrenal steroidogenesis via $\mathrm{H} 2 \mathrm{O} 2$-dependent, reversible inactivation of peroxiredoxin III in mitochondria. Mol Cell 46, 584-594 [PubMed: 22681886]

32. Dey S, Sidor A, and O'Rourke B (2016) Compartment-specific control of reactive oxygen species scavenging by antioxidant pathway enzymes. J Biol Chem 291, 11185-11197 [PubMed: 27048652]

33. Nelson KJ, Bolduc JA, Wu H, Collins JA, Burke EA, Reisz JA, Klomsiri C, Wood ST, Yammani RR, Poole LB, Furdui CM, and Loeser RF (2018) H2O2 oxidation of cysteine residues in c-Jun Nterminal kinase 2 (JNK2) contributes to redox regulation in human articular chondrocytes. J Biol Chem

34. Kawarazaki Y, Ichijo H, and Naguro I (2014) Apoptosis signal-regulating kinase 1 as a therapeutic target. Expert Opin Ther Targets 18, 651-664 [PubMed: 24660755] 
35. Yang G, Tian SJ, Luo JP, Zheng J, Sun H, and Gao YZ (2017) PRDX1 overexpression contributes to the protection on chondrocytes from IL-1 beta-induced apoptosis. International Journal of Clinical and Experimental Medicine 10, 6538-6547

36. Kim YJ, Lee WS, Ip C, Chae HZ, Park EM, and Park YM (2006) Prx1 suppresses radiationinduced c-Jun NH2-terminal kinase signaling in lung cancer cells through interaction with the glutathione S-transferase Pi/c-Jun NH2-terminal kinase complex. Cancer Res 66, 7136-7142 [PubMed: 16849559]

37. Noh YH, Baek JY, Jeong W, Rhee SG, and Chang TS (2009) Sulfiredoxin translocation into mitochondria plays a crucial role in reducing hyperoxidized peroxiredoxin III. J Biol Chem 284, 8470-8477 [PubMed: 19176523]

38. Loeser RF, Carlson CS, Del Carlo M, and Cole A (2002) Detection of nitrotyrosine in aging and osteoarthritic cartilage: Correlation of oxidative damage with the presence of interleukin-1beta and with chondrocyte resistance to insulin-like growth factor 1 . Arthritis Rheum 46, 2349-2357 [PubMed: 12355482]

39. Carlo MD Jr., and Loeser RF (2003) Increased oxidative stress with aging reduces chondrocyte survival: correlation with intracellular glutathione levels. Arthritis Rheum 48, 3419-3430 [PubMed: 14673993]

40. Gavriilidis C, Miwa S, von Zglinicki T, Taylor RW, and Young DA (2013) Mitochondrial dysfunction in osteoarthritis is associated with down-regulation of superoxide dismutase 2. Arthritis Rheum 65, 378-387 [PubMed: 23138846]

41. Blanco FJ, Rego I, and Ruiz-Romero C (2011) The role of mitochondria in osteoarthritis. Nat Rev Rheumatol 7, 161-169 [PubMed: 21200395]

42. Lopez-Otin C, Blasco MA, Partridge L, Serrano M, and Kroemer G (2013) The hallmarks of aging. Cell 153, 1194-1217 [PubMed: 23746838]

43. Bolduc JA, Collins JA, and Loeser RF (2018) Reactive oxygen species, aging and articular cartilage homeostasis. Free Radic Biol Med 
A
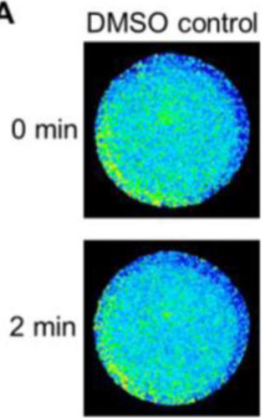

DIC

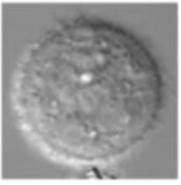

C
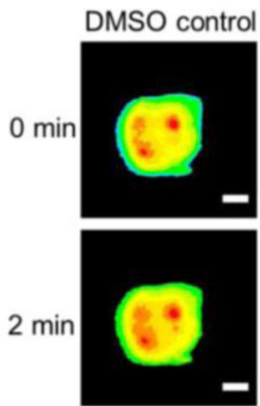

$405 n m$

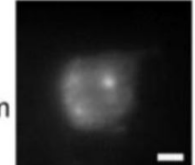

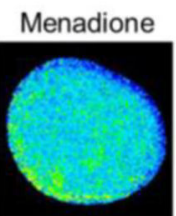
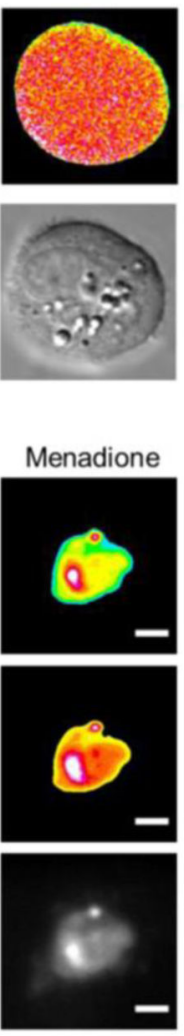
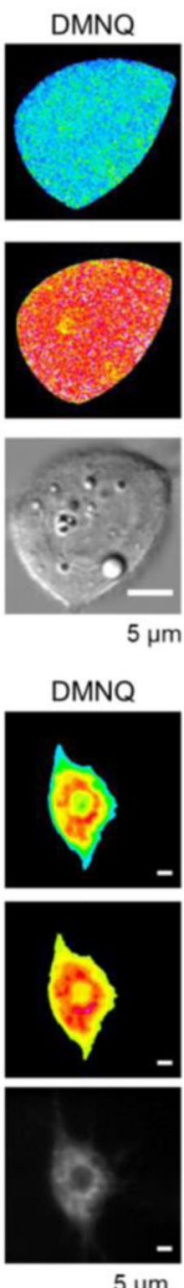

B

oxidized
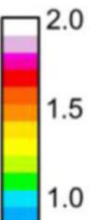

0.5

reduced

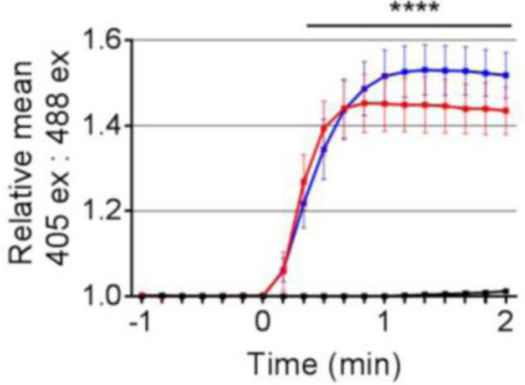

- Menadione

$\rightarrow \mathrm{DMNQ}$

$\rightarrow$ DMSO

D

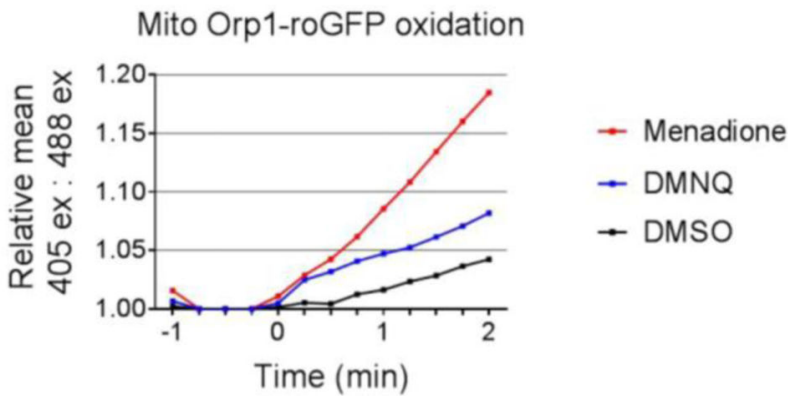

Figure 1. $\mathrm{H}_{2} \mathrm{O}_{2}$ generation in human articular chondrocytes treated with menadione and DMNQ.

The $\mathrm{H}_{2} \mathrm{O}_{2}$ redox sensor Orp1-roGFP bacolovirus was transduced into human articular chondrocytes. Cultures were treated with $25 \mu \mathrm{M}$ menadione, $25 \mu \mathrm{M}$ DMNQ, or a DMSO control. (A) Images from one representative cell for control, menadione and DMNQ treated conditions are shown in heat map format (scale bar, $5 \mu \mathrm{M}$ ). (B) Quantified data is presented as mean \pm SEM showing the relative mean 405:488 ratio from 10 individual cells taken from 3 independent donors. Asterisks represent significant differences comparing menadione and DMNQ treated chondrocytes to DMSO control (****p<0.0001) (ANOVA). To assess mitochondrial $\mathrm{H}_{2} \mathrm{O}_{2}$ generation, chondrocytes were transduced with mito-Orp1-roGFP and treated with menadione ( $25 \mu \mathrm{M})$, DMNQ $(25 \mu \mathrm{M})$, or a DMSO control. (C) Images from one representative cell for control, menadione and DMNQ treated conditions are shown in heat map format (scale bar, $5 \mu \mathrm{M}$ ). (D) Representative data from 2 independent donors (analysing 3 independent cells per donor). Presented data is relative mean 405:488 ratios from 3 individual cells taken from 1 donor. 
A

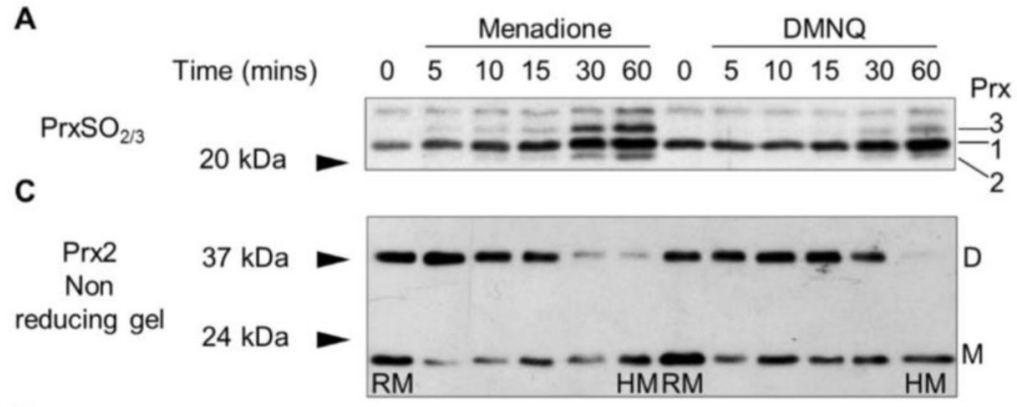

D Prx3
Non
reducing gel

$\beta$-tubulin Reducing ge

E

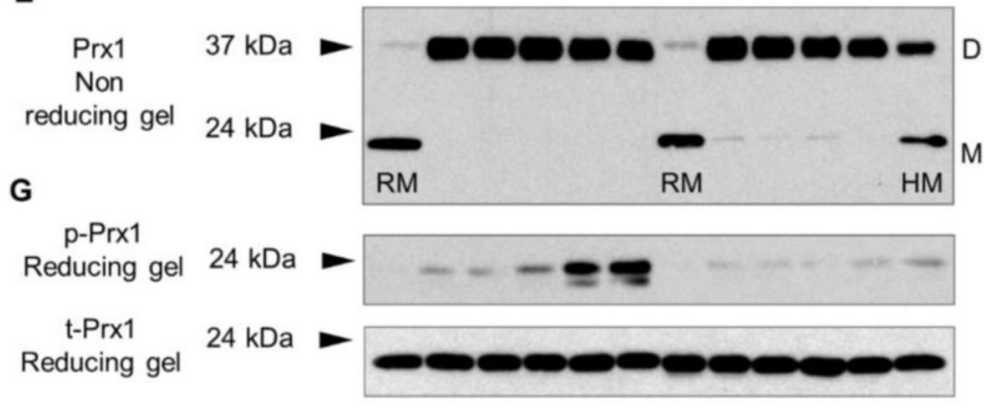

B

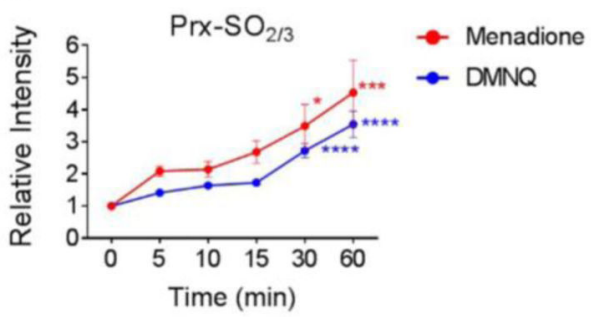

F

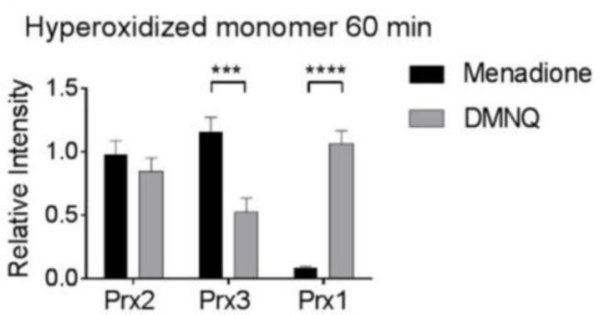

H

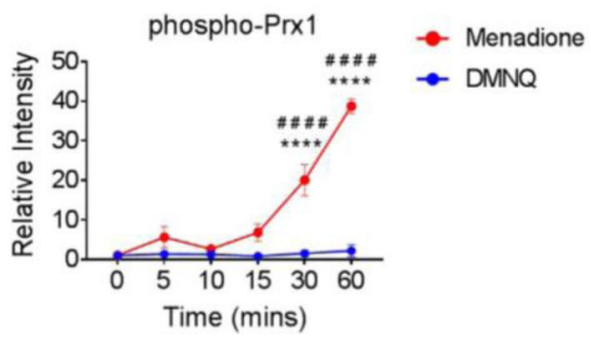

Figure 2. Prx1, Prx2 and Prx3 redox status in human articular chondrocytes in response to menadione and DMNQ-induced oxidative stress.

Confluent human articular chondrocytes were cultured in serum-free media overnight and treated for the indicated times with $25 \mu \mathrm{M}$ menadione or $25 \mu \mathrm{M}$ DMNQ. After experimental exposures, cell monolayers were incubated for $10 \mathrm{~min}$ in an alkylating buffer containing 100 $\mathrm{mM}$ NEM in order to alkylate reduced thiols. Cell lysates were then prepared in lysis buffer containing $100 \mathrm{mM}$ NEM. (A) Hyperoxidized Prxs were identified by reducing immunoblots using an antibody to $\operatorname{PrxSO}_{2 / 3}$. (B) Results of densitometric analysis from $\operatorname{PrxSO}_{2 / 3}$ immunoblots. Data are mean \pm SEM and expressed as relative intensity compared to untreated controls from $n=4$ independent experiments. Asterisks represent significant differences compared to control $(* p<0.05$, *** $p<0.001$, **** $\mathrm{p}<0.0001)$ (ANOVA). (C-E) Under non-reducing conditions, immunoblots for total Prx1, Prx2 and Prx3 allowed for identification of Prx reduced monomers (labelled RM on blots) and hyperoxidized monomers (labelled HM on blots). Prx1-Prx3 presented with an oxidized dimer (labelled D on blots) (C, D, E). (F) Densitometric comparative analysis of Prx1, Prx2 and Prx3 hyperoxidized monomers observed at 60 min treatment with menadione or DMNQ. Asterisks represent significant differences between menadione and DMNQ treatments $(* * * p<0.001, * * * * p<0.0001)$ (ANOVA) from a minimum of $\mathrm{n}=3$ independent experiments. (G) Menadione and DMNQ-induced phosphorylation of Prx1 was identified by nonreducing immunoblots using an antibody to phospho-Prx1 (Tyr194). Total Prx1 was used as 
a loading control. (H) Results of densitometric analysis from phospho-Prx1 immunoblots. Data are mean \pm SEM normalized to total-Prx 1 and expressed as relative intensity compared to untreated controls. Asterisks represent significant differences compared to control $(* * * * p<0.0001)$. \# represent significant differences between menadione and DMNQ at that time point (\#\#\# \# $p<0.0001$ ) (ANOVA). 
A

Figure 3. Effect of menadione and DMNQ-induced oxidative stress to phosphorylate proteins pertinent to IGF-1 and MAP kinase signaling cascades.

Confluent human articular chondrocytes were treated with IGF-1 (50 ng/mL), menadione $(25 \mu \mathrm{M})$, or DMNQ $(25 \mu \mathrm{M})$ for $60 \mathrm{~min}$. Cell lysates were prepared and immunoblotting was performed under reducing conditions with antibodies to phosphorylated IGF-receptor, Akt, PRAS40, p38, ERK, JNK and c-Jun. Respective total antibodies were used to assess loading controls. (A) Representative immunoblots from 3 independent experiments $(n=3)$. (B-H) Results of densitometric analysis showing phosphorylation of IGF-receptor, Akt, PRAS40, p38, ERK, JNK and c-Jun. Phospho-proteins are normalized to respective total 
protein. For c-Jun, $\beta$-actin was used to assess loading control. Arrow on c-Jun blots indicates electrophoretic shift of the c-Jun monomer which is indicative of serine and threonine phosphorylation (maximal phosphorylation). Asterisks represent significant differences compared to untreated controls $(* p<0.05, * * * p<0.001)$. 
A

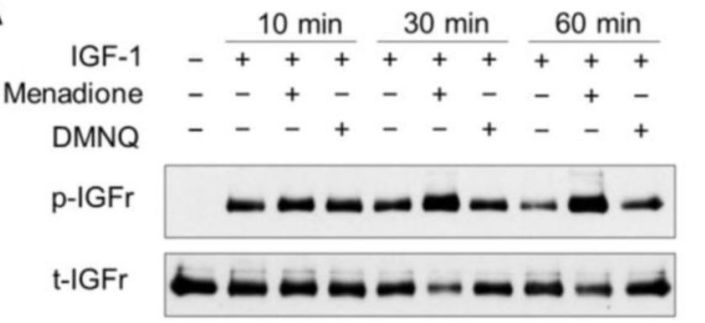

p-Akt

t-Akt

p-PRAS40

t-PRAS40

p-P38

t-P38

p-ERK

t-ERK

p-JNK

t-JNK

p-c-Jun

t-C-Jun

$\beta$-actin

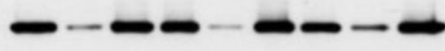

$-\infty-\infty-\infty-\infty$

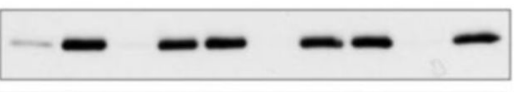

(5)
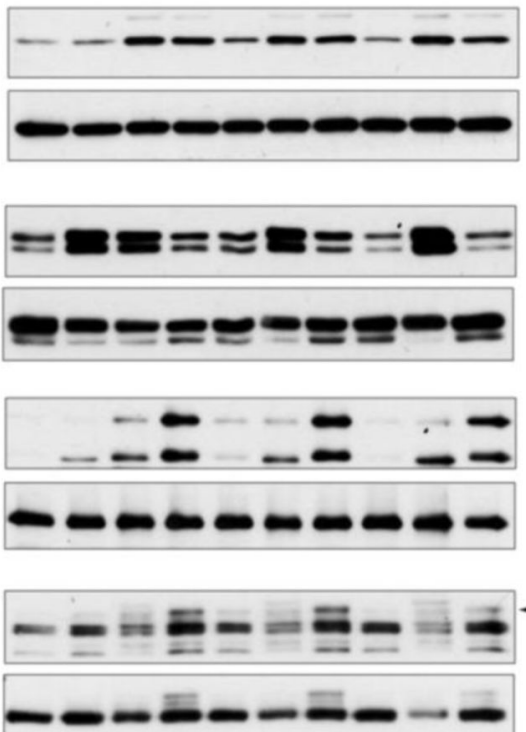

$-\infty-\infty-\infty$

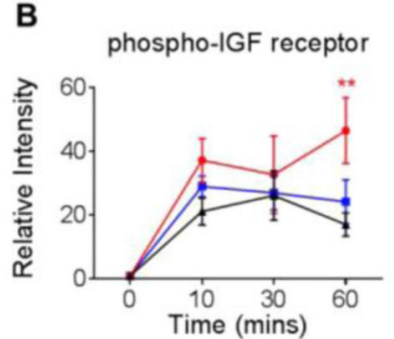

D

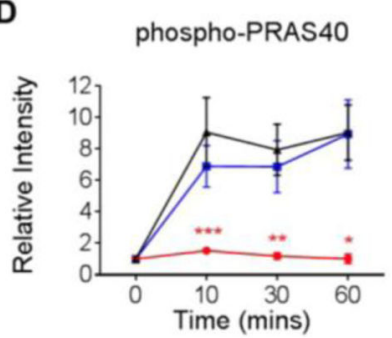

phospho-PRAS40

F

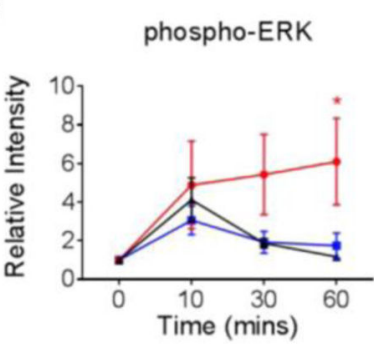

H

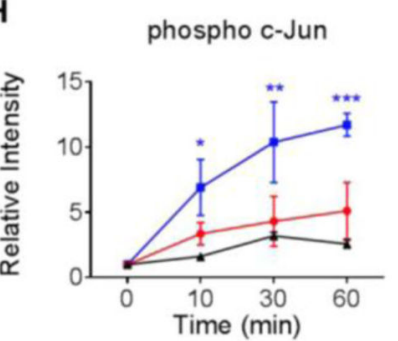

DMNQ+IGF-1

C

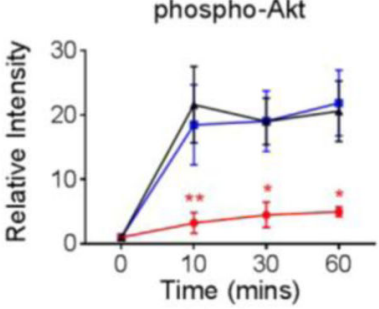

E

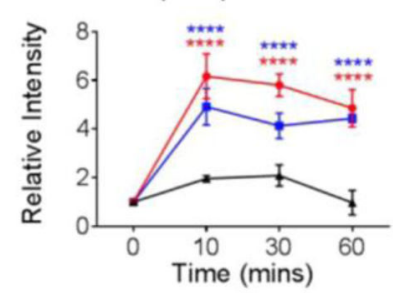

G

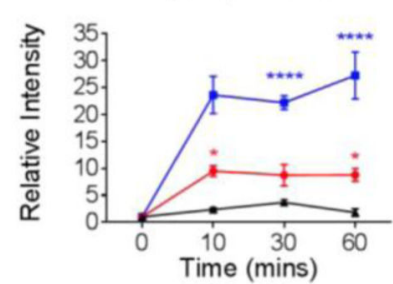

I

Cell viability

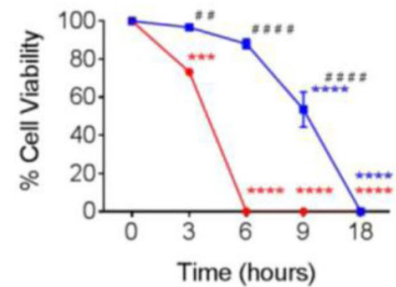

Figure 4. Effect of menadione and DMNQ to differentially regulate IGF-1 signaling. Human articular chondrocytes were treated with IGF-1 $(50 \mathrm{ng} / \mathrm{ml})$ alone or pre-treated with menadione $(25 \mu \mathrm{M})$ or DMNQ $(25 \mu \mathrm{M})$ for 30 min prior to IGF-1 treatment for the indicated times. Cell lysates were prepared and immunoblotted under reducing conditions with antibodies to phosphorylated IGF-receptor, Akt, PRAS40, p38, ERK, JNK and c-Jun. Respective total antibodies were used to assess loading controls. (A) Representative immunoblots from 3 independent experiments $(n=3)$. (B-H) Results of densitometric analysis showing phosphorylation of IGF-receptor, Akt, PRAS40, p38, ERK, JNK and cJun. Phosphoproteins were normalized to respective total protein. For c-Jun, $\beta$-actin was used to assess loading control. Arrow on c-Jun blots indicates electrophoretic shift of the cJun monomer which is indicative of serine and threonine phosphorylation (maximal 
phosphorylation). Asterisks represent significant differences compared to IGF-1 at that time point $* p<0.05, * * p<0.01, * * * p<0.001, * * * * p<0.0001$ (ANOVA). (I) Chondrocytes were treated with menadione or DMNQ for 18 hours and percentage cell viability was quantified $($ mean \pm SEM) and compared to untreated control values $(n=3)$. Asterisks represent significant differences compared to controls values $(* * p<0.01, * * * * p<0.0001)$ (ANOVA). \# represent significant differences between menadione and DMNQ at that time point (\# $p<0.01$, \#\# \# $p<0.0001$ ) (ANOVA). 

levels. $\mathrm{PrxSO}_{2 / 3}$ formation. (D) Effect of adenoviral overexpression of Prx 2 and Prx 3 on
A

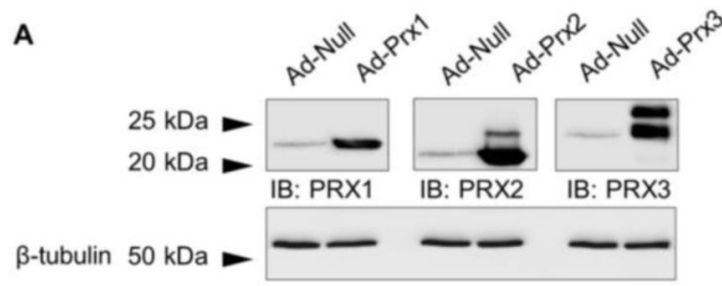

B

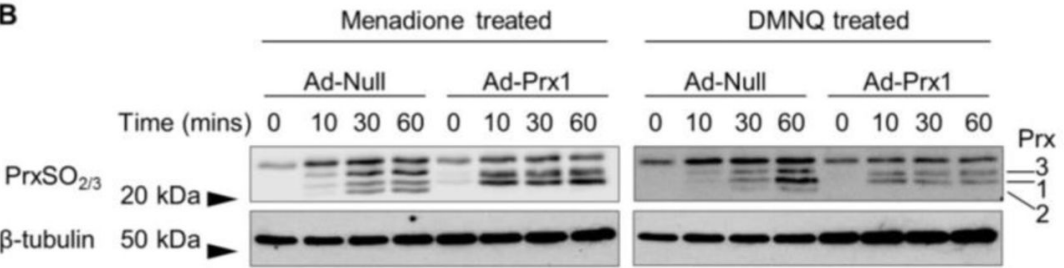

D
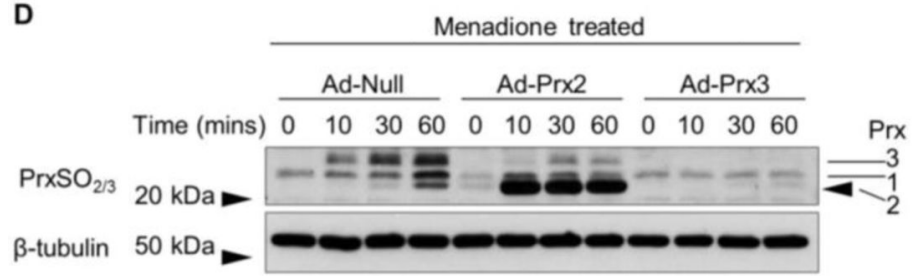

F DMNQ treated

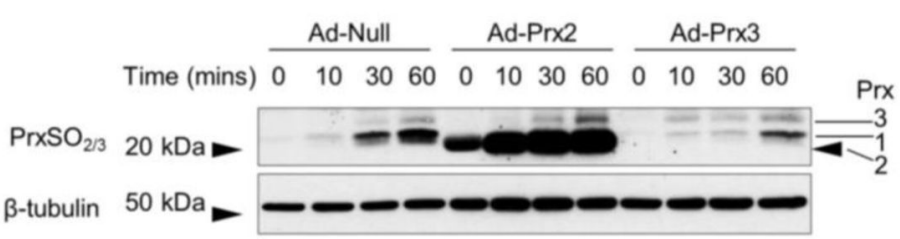

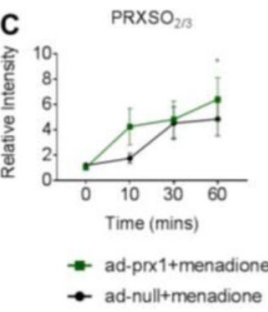

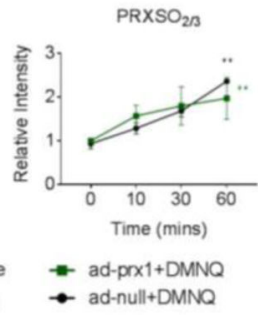

E
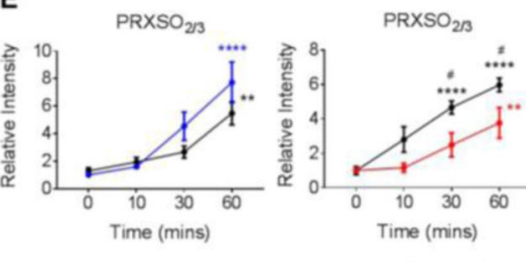

- ad-Prx2+menadione

- ad-null+menadione

- ad-Prx3+menadione

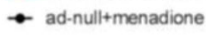

G

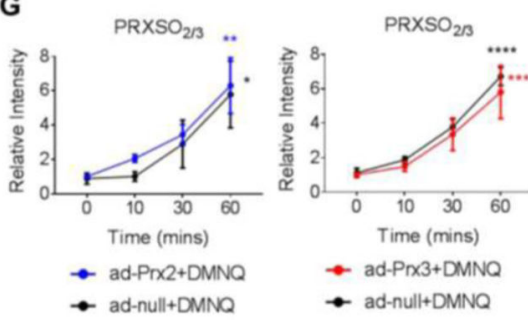

Figure 5. Effect of adenoviral overexpression of Prx1, Prx2 and Prx3 on Prx hyperoxidation

Human articular chondrocytes were transduced with adenoviral vectors encoding Prx1, Prx2, Prx3 or a null empty vector control and treated with menadione $(25 \mu \mathrm{M})$ or DMNQ $(25 \mu \mathrm{M})$ for the indicated times. (A) Lysates from unstimulated chondrocytes transduced with Prx1-3 adenovirus or null empty control were immunoblotted with antibodies to Prx1-Prx3 or $\beta$ tubulin as a protein loading control. (B) Effect of Prx 1 overexpression on menadione and DMNQ-induced $\operatorname{PrxSO}_{2 / 3}$ formation. Representative immunoblots from 7 independent experiments $(n=7)$ with menadione treatment and 4 independent experiments $(n=4)$ with DMNQ treatment. (C) Results of densitometric analysis from $\operatorname{PrxSO}_{2 / 3}$ immunoblots showing the effect of adenoviral overexpression of Prx 1 on menadione and DMNQ-induced menadione-induced $\mathrm{PrxSO}_{2 / 3}$ formation. Representative immunoblots from 6 independent experiments $(n=6)$. (E) Results of densitometric analysis from $\operatorname{PrxSO}_{2 / 3}$ immunoblots showing the effect of adenoviral overexpression of Prx 2 and Prx 3 on menadione induced $\mathrm{PrxSO}_{2 / 3}$ formation. (F) Effect of adenoviral overexpression of Prx 2 and Prx3 on DMNQinduced $\mathrm{PrxSO}_{2 / 3}$ formation. Representative immunoblots from 4 independent experiments 
$(\mathrm{n}=4)$. (G) Results of densitometric analysis from $\mathrm{PrxSO}_{2 / 3}$ immunoblots showing the effect of adenoviral overexpression of Prx 2 and Prx 3 on DMNQ induced $\operatorname{PrxSO}_{2 / 3}$ formation. All data are mean \pm SEM normalized to untreated adenoviral control values. Asterisks represent significant differences compared to controls values $* p<0.05, * * p<0.01, * * * p<0.001$, $* * * * p<0.0001)$ (ANOVA). \# represent significant differences between Prx overexpressing vector and null empty vector at that time point (\# $p<0.05$ ) (ANOVA). 
A

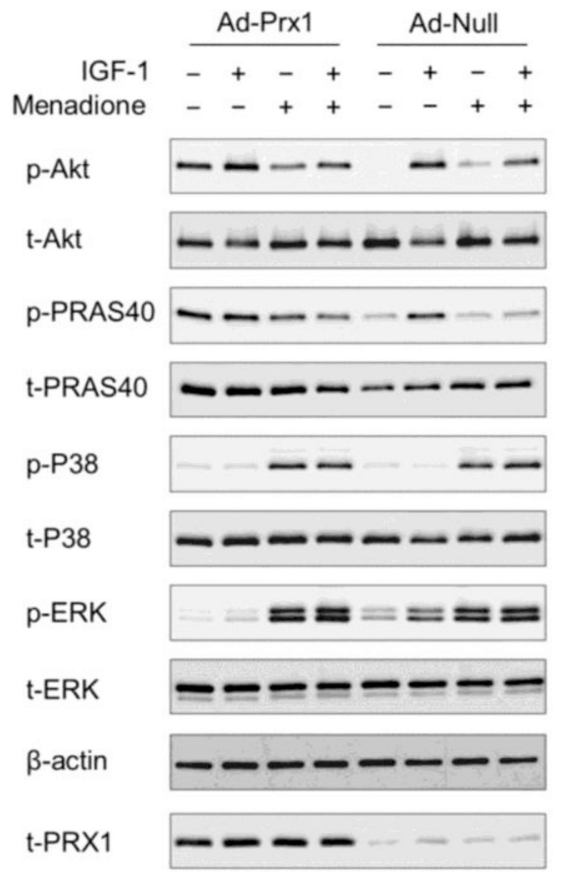

F

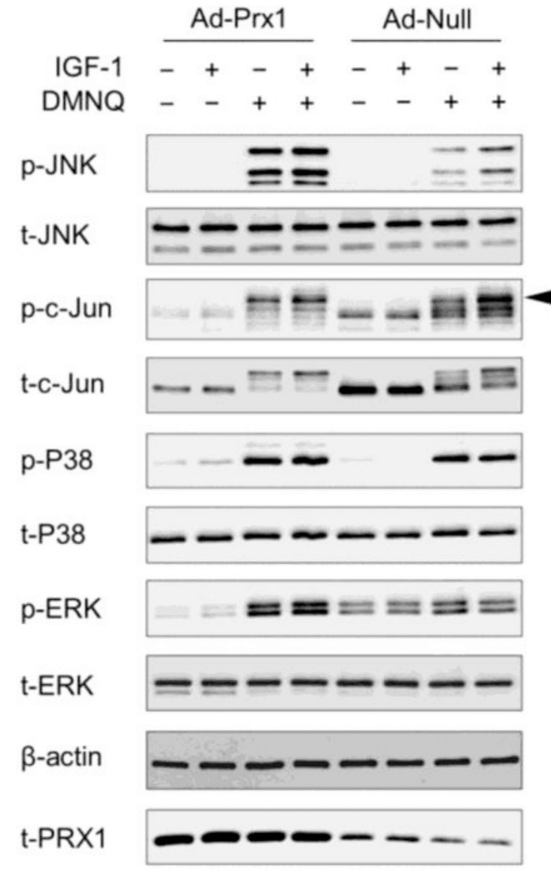

ad-PRX1 $\square$ Null vector

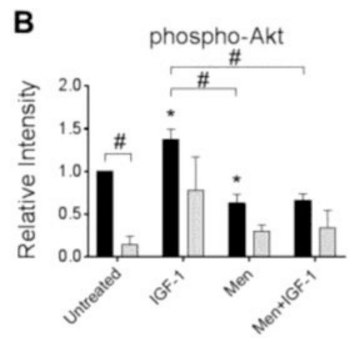

D

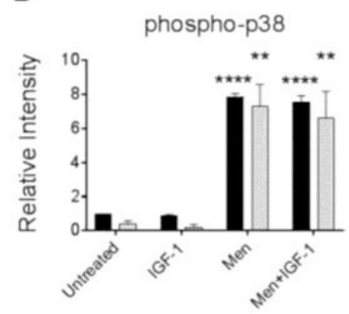

C

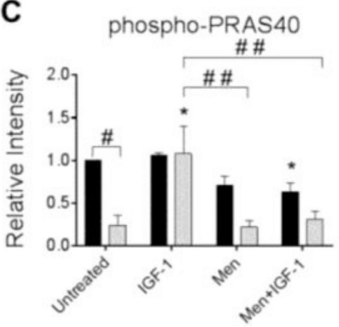

E

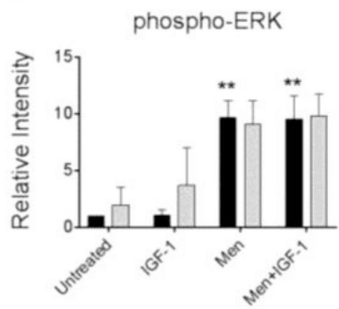

G

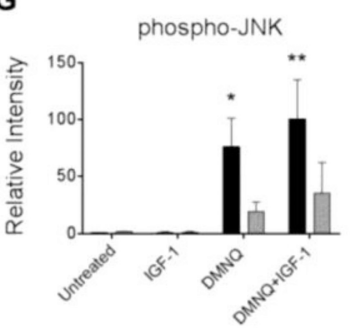

I

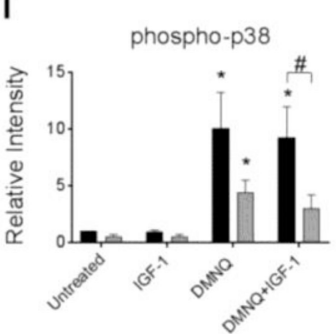

H

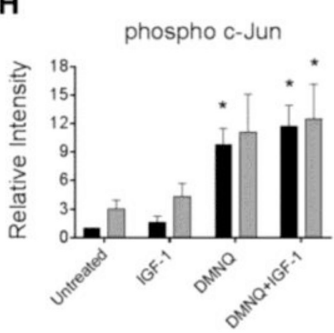

J phospho-ERK

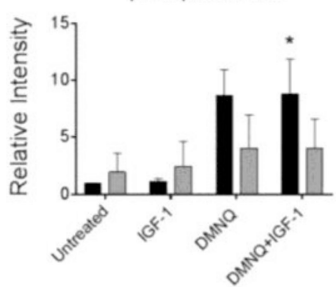

Figure 6. Effect of adenoviral overexpression of Prx1 on IGF-1 and MAP kinase signaling with and without menadione and DMNQ.

Chondrocytes were transduced with adenoviral vectors encoding Prx1 or a null empty vector control and were treated with IGF-1 alone ( $50 \mathrm{ng} / \mathrm{ml})$, menadione $(25 \mu \mathrm{M})$, DMNQ $(25 \mu \mathrm{M})$ or pre-treated with menadione $(25 \mu \mathrm{M})$ or DMNQ $(25 \mu \mathrm{M})$ for $30 \mathrm{~min}$ prior to IGF-1 treatment for $60 \mathrm{~min}$. Cell lysates were immunoblotted with antibodies to the indicated proteins pertinent to IGF-1 and MAP kinase signaling cascades. (A) Representative immunoblots from 3 independent experiments $(n=3)$ showing the effect of Prx 1 overexpression on menadione-induced phosphorylation of Akt, PRAS40, p38, and ERK. (BE) Results of densitometric analysis showing the effect of Prx1 overexpression on 
phosphorylation of Akt, PRAS40, p38, and ERK in response to menadione treatment. (F) Representative immunoblots from 4 independent experiments $(n=4)$ showing the effect of Prx1 overexpression on DMNQ-induced phosphorylation of JNK, c-Jun, p38, and ERK. (GJ) Results of densitometric analysis showing the effect of Prx 1 overexpression on phosphorylation of JNK, c-Jun, p38, and ERK in response to DMNQ treatment. Phosphoproteins are normalized to respective total protein. For c-Jun, $\beta$-actin was used as a loading control. Arrow on c-Jun blots indicates electrophoretic shift of the c-Jun monomer which is indicative of serine and threonine phosphorylation (maximal phosphorylation). Data are mean \pm SEM expressed as relative intensity compared to ad-Prx1 control Asterisks represent significant differences compared to control $(* p<0.05$, $* * p<0.01$, **** $p<0.0001)$. \# represent significant differences between highlighted conditions (\# $p<0.05$, \# \# $p<0.01$ ) (ANOVA). 
A

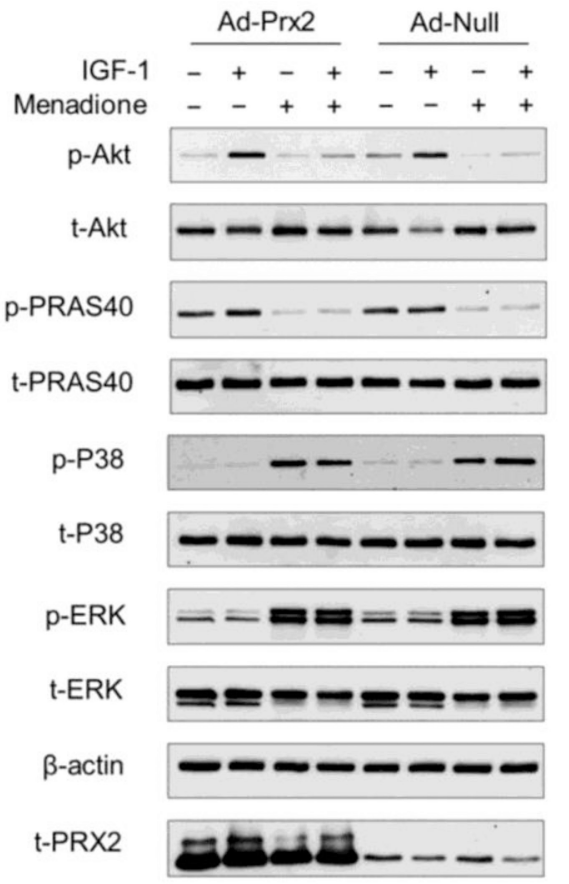

F

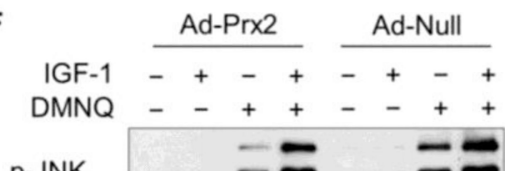

p-JNK

t-JNK

p-c-Jun

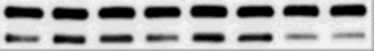

t-c-Jun

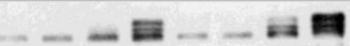

p-P38

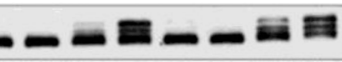

t-P38

p-ERK
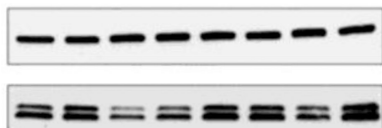

t-ERK

$\beta$-actin

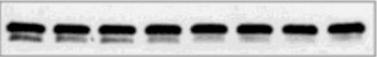



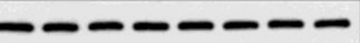

t-PRX2

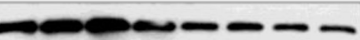

ad-PRX2 $\square$ Null vector
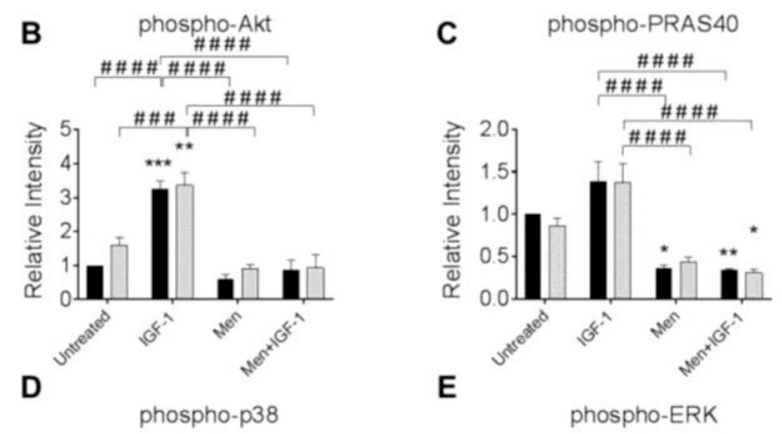

E

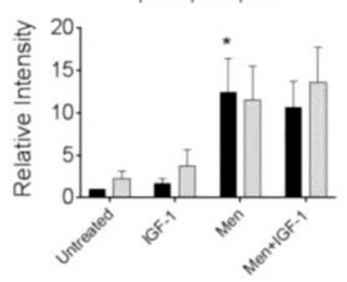

G

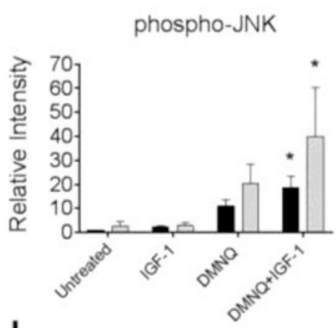

phospho-p38

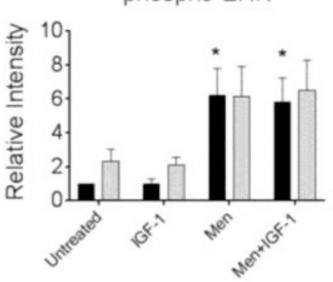

H

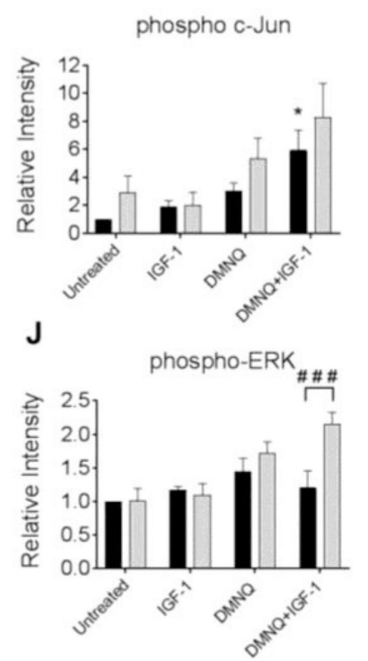

Figure 7. Effect of adenoviral overexpression of Prx2 on IGF-1 and MAP kinase signaling with and without menadione and DMNQ.

Chondrocytes were transduced with an adenoviral vector encoding Prx 2 or a null empty vector control and were treated with IGF-1 alone $(50 \mathrm{ng} / \mathrm{ml})$, menadione $(25 \mu \mathrm{M})$, DMNQ $(25 \mu \mathrm{M})$ or pre-treated with menadione $(25 \mu \mathrm{M})$ or DMNQ $(25 \mu \mathrm{M})$ for 30 min prior to IGF-1 treatment for $60 \mathrm{~min}$. Cell lysates were immunoblotted with antibodies to proteins pertinent to IGF-1 and MAP kinase signaling cascades. (A) Representative immunoblots from 3 independent experiments $(n=3)$ showing the effect of Prx 2 overexpression on menadione-induced phosphorylation of Akt, PRAS40, p38, and ERK. (B-E) Results of densitometric analysis showing the effect of Prx 2 overexpression on phosphorylation of Akt, 
PRAS40, p38, and ERK in response to menadione treatment. (F) Representative immunoblots from 5 independent experiments $(n=5)$ showing the effect of Prx2 overexpression on DMNQ-induced phosphorylation of JNK, c-Jun, p38, and ERK. Arrow on c-Jun blots indicates electrophoretic shift of the c-Jun monomer which is indicative of serine and threonine phosphorylation (maximal phosphorylation). (G-J) Results of densitometric analysis showing the effect of Prx2 overexpression on phosphorylation of JNK, c-Jun, p38, and ERK in response to DMNQ treatment. Phospho-proteins are normalized to respective total protein. For c-Jun, $\beta$-actin was used as a loading control. Data are mean \pm SEM expressed as relative intensity compared to ad-Prx 2 control. Asterisks represent significant differences compared to control $(* p<0.05, * * p<0.01, * * * p<0.001)$. \# represent significant differences between highlighted conditions (\# \# \# $p<0.001$, \# \# \# $p<0.0001$ ) (ANOVA). 
A

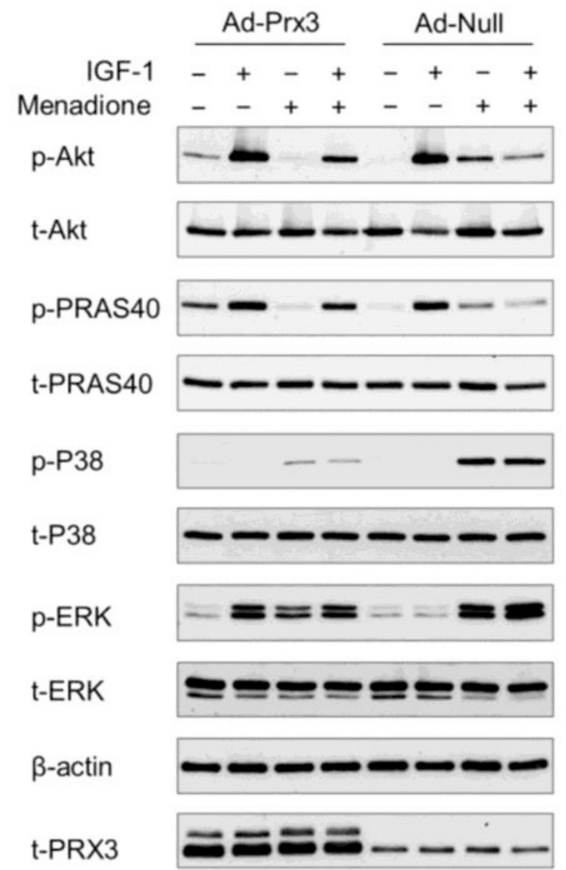

F

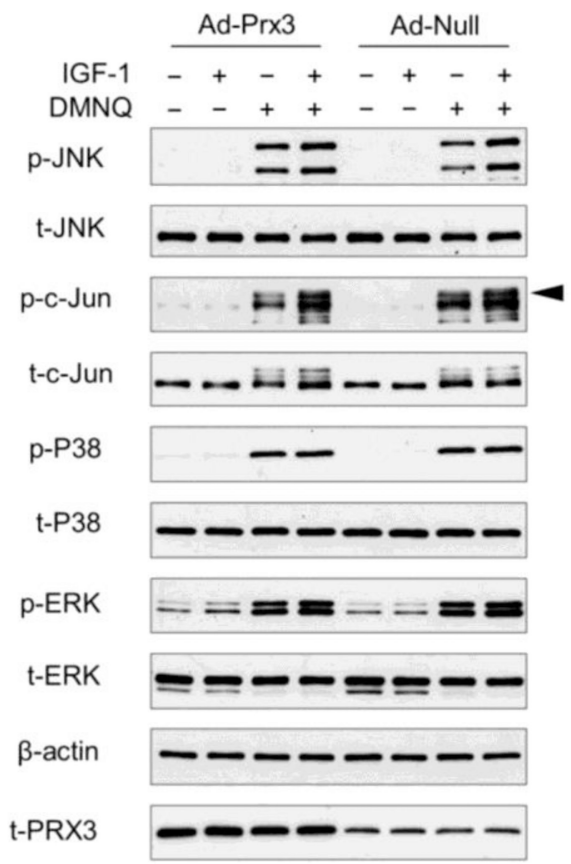

B

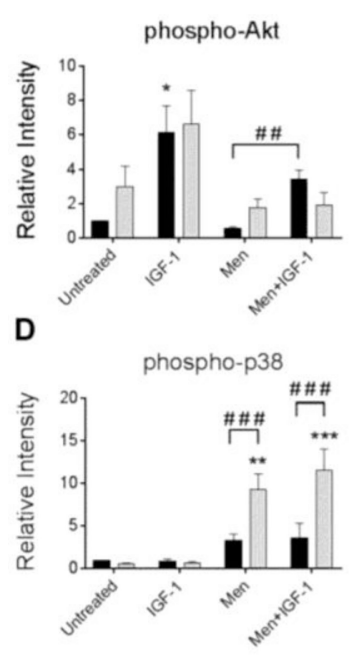

C

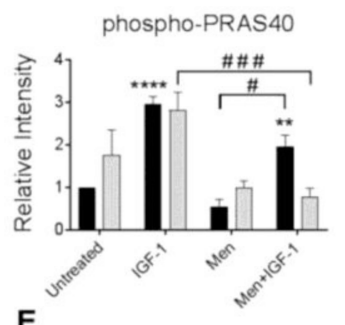

E

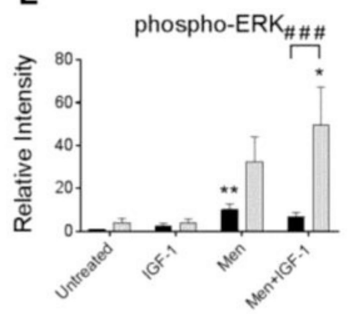

ad-PRX3 口 Null vector

G

H
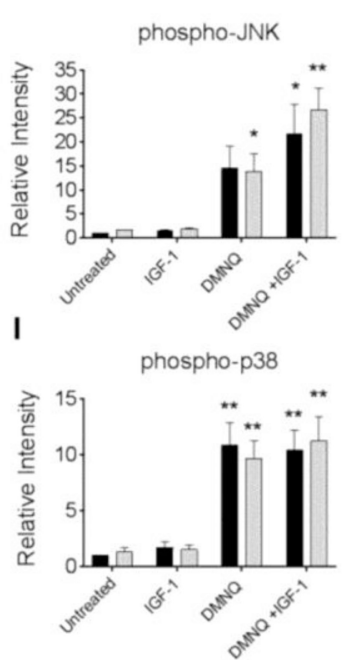

H
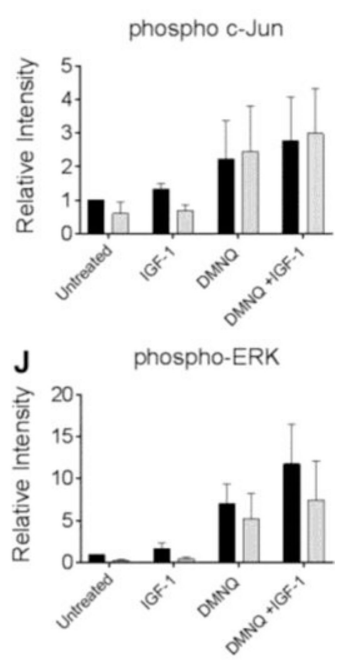

Figure 8. Effect of adenoviral overexpression of Prx3 on IGF-1 and MAP kinase signaling with and without menadione and DMNQ.

Chondrocytes were transduced with an adenoviral vector encoding Prx3 or a null empty vector control and were treated with IGF-1 alone $(50 \mathrm{ng} / \mathrm{ml})$, menadione $(25 \mu \mathrm{M})$, DMNQ $(25 \mu \mathrm{M})$ or pre-treated with menadione $(25 \mu \mathrm{M})$ or DMNQ $(25 \mu \mathrm{M})$ for 30 min prior to IGF-1 treatment for $60 \mathrm{~min}$. Cell lysates were immunoblotted with antibodies to proteins pertinent to IGF-1 and MAP kinase signaling cascades. (A) Representative immunoblots from 4 independent experiments $(n=4)$ showing the effect of Prx 3 overexpression on menadione-induced phosphorylation of Akt, PRAS40, p38, and ERK. (B-E) Results of densitometric analysis showing the effect of Prx3 overexpression on phosphorylation of Akt, 
PRAS40, p38, and ERK in response to menadione treatment. (F) Representative immunoblots from 3 independent experiments $(n=3)$ showing the effect of Prx3 overexpression on DMNQ-induced phosphorylation of JNK, c-Jun, p38, and ERK. (G-J) Results of densitometric analysis showing the effect of Prx2 overexpression on phosphorylation of JNK, c-Jun, p38, and ERK in response to DMNQ treatment. Phosphoproteins are normalized to respective total protein. For c-Jun, $\beta$-actin was used as a loading control. Arrow on c-Jun blots indicates electrophoretic shift of the c-Jun monomer which is indicative of serine and threonine phosphorylation (maximal phosphorylation). Data are mean \pm SEM expressed as relative intensity compared to ad-Prx 3 control. Asterisks represent significant differences compared to control $(* p<0.05, * * p<0.01, * * * p<0.001)$. \# represent significant differences between highlighted conditions (\# $p<0.05$, \# \# $p<0.01$, \# \# \# $p<0.001$ ) (ANOVA). 